The quality of this microform is heavily dependent upon the quality of the original thesis submitted for microfilming. Every effort has been made to ensure the higinest quality of reproduction possible.

If pages are missing, contact the university which granted the degree.

Some pages may have indistinct print especially if the original pages were typed with a poor typewriter ribbon or if the university sent us an inferior photocopy.

Reproduction in full or in part of this microform is governed by the Canadian Copyright Act, R.S.C. 1970, c. C-30, and subsequent amendments.
La qualité de cette microforme dépend grandement de la qualité de la thèse soumise au microfilmage. Nous avons tout fait pour assurer une qualité supérieure de reproduction.

S'il manque des pages, veuillez communiquer avec 'université qui a conféré le grade.

La qualité d'impression de certaines pages peut laisser à désirer, surtout si les pages originales ont été dactylographiées à l'aide d'un ruban usé ou si l'université nous a fait parvenir une photocopie de qualité inférieure.

La reproduction, même partielle, de cette microforme est soumise à la Loi canadienne sur le droit d'auteur, SRC 1970, c. C-30, et ses amendements subséquents. 


\title{
DECENTRALIZED IMPEDANCE CONTROL
}

\author{
Michel Doyon \\ B. ing. Universitć Laval 1992 \\ Department of Electrical Engineering \\ McGill University, Montréal
}

A thesis submitted to the Faculty of Graduate Studies and Research in partial fulfillment of the requirements for the degree of Master of Engineering

September 1994

(C) Michel Doyon 
National Library

of Canada

Acquisitions and

Bibliographic Services Branch

395 Wellington Street

Onawa. Onlario

KIA ONA
Bibliotheque nationale

du Canada

Direction des acquisitions et

des services bibliographiques

395. rue Wellington

Ottawa (Ontario)

KIA ON4
Your sin botreteloremese

Our ine intiposferemie
THE AUTHOR HAS GRANTED AN IRREVOCABLE NON-EXCLUSIVE LICENCE ALLOWING THE NATIONAL LIBRARY OF CANADA TO

REPRODUCE, LOAN, DISTRIBUTE OR SELL COPIES OF HIS/HER THESIS BY ANY MEANS AND IN ANY FORM OR FORMAT, MAKING THIS THESIS

AVAILABLE TO INTERESTED PERSONS.
L'AUTEUR A ACCORDE UNE LICENCE IRREVOCABLE ET NON EXCLUSIVE PERMETTANT A LA BIBLIOTHEQUE NATIONALE DU CANADA DE REPRODUIRE, PRETER, DISTRIBUER OU VENDRE DES COPIES DE SA THESE DE QUELQUE MANIERE ET SOUS QUELQUE FORME QUE CE SOIT POUR METTRE DES EXEMPLAIRES DE CETTE THESE A LA DISPOSITION DES PERSONNE INTERESSEES.
THE AUTHOR RETAINS OWNERSHIP OF THE COPYRIGHT IN HIS/HER THESIS. NEITHER THE THESIS NOR SUBSTANTIAL EXTRACTS FROM IT MAY BE PRINTED OR OTHERWISE REPRODUCED WITHOUT HIS/HER PERMISSION.
L'AUTEUR CONSERVE LA PROPRIETE DU DROIT D'AUTEUR QUI PROTEGE SA THESE. NI LA THESE NI DES EXTRAITS SUBSTANTIELS DE CELLECI NE DOIVENT ETRE IMPRIMES OU AUTREMENT REPRODUITS SANS SON AUTORISATION.

ISBN $\quad 0-315-99962-4$

\section{Canadä̀}




\section{Abstract}

Many applications of manipulators to date have been based on position control, but when a robot manipulator makes contact with the environment, the control of force and position is required. Onc approach, impedance control, is to control the manipulator such that the relationship between position and force is well defined. For cxample, such that the robot behaves like a mass-spring-damper system whose parameters can be specified arbitrarily. In the recent years, many of the impedance control implementations were using force feedback from an end-effector force sensor and the impedance control was performed by forming the error in Cartesian coordinates. The implementation proposed here requires instead co-located torque sensors and the feedback control signals are based solely on joint variables, torque and displacement. Co-located torque sensors lead to a larger control bandwidth since the structural dynamics of the arm is seen as a perturbation, but is not part of the plant to be controlled. Simple SISO controllers, designed to modulate individual joint impedances, can achieve diagonal (decoupled) impedance matrices in Cartesian coordinates, provided that a set of SISO compensators, called here "decouplers", are set up to cancel the non-linear coupling among the joints. This strategy results in a simple computational architecture which does not require complex coordinates transformations to be performed at servo rate. The method can be used with nonredundant and redundant manipulators and experimental results are discussed using a seven DOF manipulator available at the Institut de recherche d'Hydro-Québec. 


\section{Résumé}

La plupart des applications robotiques utilisées jusqu à maintenant, utilisalent la commande de position. Cependant. quand un manipulateur effectue des tâches impliquant des contacts avec lenvironnement, la force exercéc doit aussi ittre prise en compte. Une approche consiste à permettre au robot de réagir tel un systieme masseamortisseur-ressort permettant ainsi une relation dynamique entre la position et la force exercée par le robot. C'est ce qu'on appelle la commande d'impédance.

Au cours des dernière années, la plupart des lois de commande dimpédance utilisaient un capteur de force placé à l'extrémité du robot ct la commande d'impédance était faite à haut niveau dans l'espace cartćsien. L̈implantation que nous proposons ne requière que des capteurs de couple localisés au niveau des actionneurs et les signaux de commande ne dépendent que des variables de l'espace des joints, le couple et la position angulaire. L'utilisation de capteurs de couple à chaque actionneur permet. d'obtenir une bande passante supéricure car la dynamique structurelle du robot est. perçue comme une perturbation et n'est pas incluse dans la boucle de commande. De simple compensateurs SISO modulant l'impédance des articulations auxquels on ajoute d'autres compensateurs SISO permettant d'annuler le couplage non linéaire entre les articulations assurent l'obtention d'une impédance découpléc en coordonnées cartésiennes. Cette loi de commande fonctionne avec des robot redondants ou non, et ses performances ont été vérifiées expérimentalement en utilisant un manipulatcur à sept degrés de liberté situé à l'Institut de recherche d'Hydro-Québec. 


\section{Acknowledgements}

I would like to take this opportunity to thank the people who helped me during my graduate studies. I wish to express my gratitude to my parents who always believed in me and supported me, and to my wife Linda for her constant encouragements. Next, my thanks go to my supervisor, Vincent Hayward, for his instruction, guidance. encouragements and for generally doing more than he had to throughout the work of this thesis.

Mes remerciements vont ensuite aux gens de l'Institut de recherche d'HydroQuébec (IREQ). Je voudrais plus particulièrement remercier Michel Pelletier, qui a en quelque sorte agit comme co-superviseur de mes travaux à l'IREQ, pour ses conseils et multiples recommandations de même que Pierre Girard qui m'a permis de faire mes études graduées en collaboration avec la division robotique de l'Institut de recherche d'Hydro-Québec et qui a mis à ma disposition tout le support administratif et technique nécéssaire au bon déroulement de mes travaux. Je tiens aussi à remercier Martin Boyer, Régis Houde et George Osmolski pour leur aide et valeureux conseils spécialement lors de l'implantation de l'algorithme de contrôle d'impédance décentralisée sur le robot Sarcos.

The financial support for this work was provided in part by the Natural Science and Engineering Research Council, in the form of a graduate scholarship and in part by the Institut de recherche d'Hydro-Québec. Their support is gratefully acknowledged. 


\section{Contents}

Abstract $\quad$ ii

Résumé

Acknowledgements iv

1 Introduction 1

2 Literature Review 3

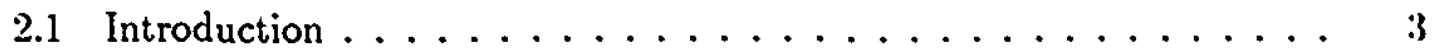

2.2 Constrained and Unconstrained Tasks . . . . . . . . . . . . I

2.2 .1 Unconstrained tasks ................. 4

2.2 .2 Constrained tasks . . . . . . . . . . . . . . 4

2.3 Hybrid control . . . . . . . . . . . . . . . . j

2.3 .1 Discussion .......................

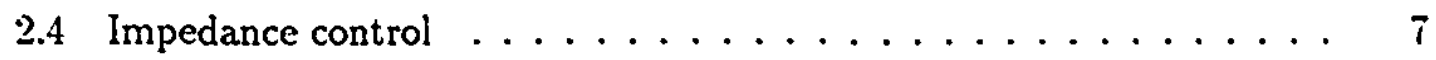

2.4.1 Position-based impedance control ............. 12

2.4.2 Torque-based impedance control ............. 14

2.5 Decentralized control . . . . . . . . . . . . . . 16

2.6 Other methods $\ldots \ldots \ldots \ldots \ldots \ldots \ldots$ 
3 Decentralized control of impedance 18

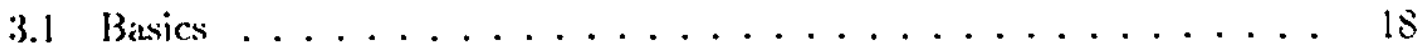

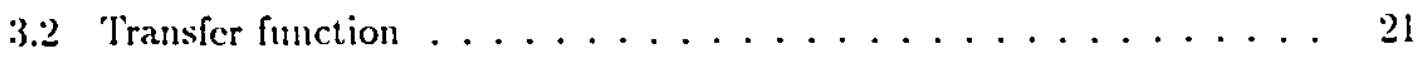

3.3 Continuous Time Stability . . . . . . . . . . . $2 \cdot 4$

3.4 Discrete Time System Stability . . . . . . . . . . . . 26

3.4 .1 Coupled multi variable case .................. 2s

3.5 Controllability and Observability ............. 30

3.6 Redundant robots . . . . . . . . . . . . . . 32

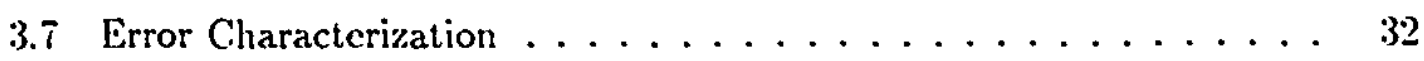

3.T.1 Error on the stiffness tern . ............ 32

3.7 .2 Error on the Damping term .......................

3.5 Use of the Redundancy of Robots . . . . . . . . . . . . 39

3.9 Reachable Impedance . . . . . . . . . . . . . . . 41

3.9 .1 Unmodelled Flexibility ................ 41

3.9 .2 Sampling Period .................... 42

3.9.3 PD Controllers Maximum Gain ............... 42

3.9.4 Maximum Torque . . . . . . . . . . . . . . 43

3.9.5 Robot's Position .................. 43

3.10 Simulation ........................ 43

3.10 .1 Steady State Response ................. 43

3.10.2 Transient Response . . . . . . . . . . . . . . . 44

4 Manipulator dynamics $\quad 48$

4.1 Cartesian Inertia Matrix .................. 48

4.2 Gravity Compensation .................... 50

5 Experimental Results $\quad \mathbf{5 2}$

5.1 Experimental Setup . . . . . . . . . . . . . . . 52 
5.2 Implementation $\ldots \ldots \ldots \ldots \ldots \ldots \ldots \ldots \ldots \ldots \ldots$

5.3 Steady State Response . . . . . . . . . . . . . . . 53

5.4 Transient Response . . . . . . . . . . . . . . . . 5 .

5.5 Contact Tasks . . . . . . . . . . . . . 6.3

$\begin{array}{lll}6 \text { Conclusion } & 65\end{array}$

A Kinematics 67

A.1 Forward Kinematics $\ldots \ldots \ldots \ldots \ldots \ldots$ bi

A.1.1 Denavit-Hartenberg Parameters . . . . . . . . . 67

A.1.2 Transformation matrices . . . . . . . . . . 6 ii

A.2 Jacobian . . . . . . .

A.2.1 Jacobian Extension . . . . . . . . . . i3

A.3 Inverse Kinematics $\ldots \ldots \ldots \ldots \ldots \ldots$. . . . . . . .

B Calibration $\quad 77$

B.1 Proportional and Derivative controller gains $\ldots \ldots \ldots \ldots \ldots$ it

B.1.1 Proportional term ................ is

B.1.2 Derivative term . . . . . . . . . . . 83

B.2 From Joint Torques to Cartesian Forces . . . . . . . . . 86 


\section{List of Tables}

4.1 Mass estimate . . . . . . . . . . . . . . . . . 19

5.1 Desired stiffness error . . . . . . . . . . . . . 55

A.1 Slave arm Denavit-Hartenberg parameters .......... $6 \mathrm{~s}$

A.2 Sarcos Slave arm: Joint limits . . . . . . . . . . . . T0

A.3 Correspondance between DH and SRC ............ T0

B.1 Controller Proportional term calibration ........... is

B.2 Controller Derivative term calibration . . . . . . . . . . \$.5 


\section{List of Figures}

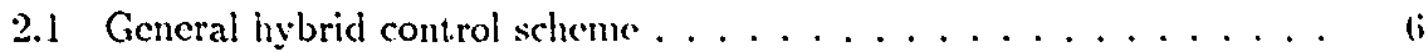

2.2 General impedance control scheme . . . . . . . . . . !

2.3 Robot and environment system modeled with linear impedances . . . 10

2.4 Position-based impedance control scheme . . . . . . . . I:

2.5 Torque-based impedance control . . . . . . . . . . . 1.5

2.6 Parametric uncertainty . . . . . . . . . . . I6

3.1 Analog and Digital Systems . . . . . . . . . . . 2

3.2 A block diagram representation $\ldots \ldots \ldots \ldots \ldots \ldots \ldots$

3.3 Mass matrix evolution is redundant degree of freedom . . . . . . 10

3.4 Optimization criteria vs redundant degree of frecelom . . . . . . . 10

3.5 Small deflection $\ldots \ldots \ldots \ldots \ldots \ldots \ldots \ldots \ldots \ldots$

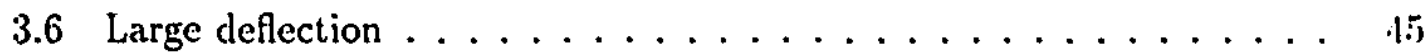

3.7 Step in $\mathrm{X}$ direction $\ldots \ldots \ldots \ldots \ldots \ldots \ldots \ldots$

3.8 Step in $Y$ direction $\ldots \ldots \ldots \ldots \ldots \ldots \ldots \ldots \ldots$

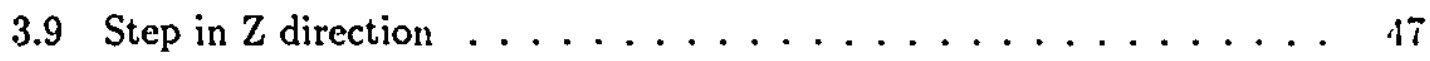

4.1 Precision vs number of parameters $\ldots \ldots \ldots \ldots \ldots \ldots$

4.2 Gravity compensation . . . . . . . . 1

5.1 The Sarcos GRLA arm . . . . . . . . . . . . . 533

5.2 Steady state with decoupler, $1000 \mathrm{~N} / \mathrm{m} \ldots \ldots \ldots \ldots \ldots$

5.3 Steady state with decoupler, $5000 \mathrm{~N} / \mathrm{m} \ldots \ldots \ldots \ldots$ 
5.1 Steady state with decoupler $10000 \times / \mathrm{m} \ldots \ldots \ldots$

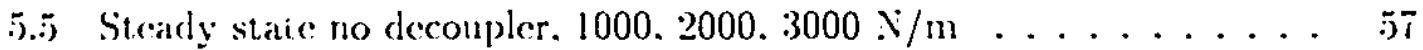

5 S.6 Steady state no crror correction. 1000. $2000.3000 \mathrm{~N} / \mathrm{m} \ldots . . .57$

5.7 Stendy state with decoupler. 1000, 2000. $3000 \mathrm{~N} / \mathrm{m} \ldots \ldots . \ldots$

5.8 Transient Response . . . . . . . . . . . . . . 59

5.9 Transient Response . . . . . . . . . . . . . . 60

5.10 Transient Responsc . . . . . . . . . . . . . . 60 60

5.11 Transient Responsc ................... 61

5.12 Transient Responsc . . . . . . . . . . . . . . 6 61

5.13 Transient Responsc ..................... 62

5.14 Contact task . . . . . . . . . . . . . . . 6 64

B.1 Analog Joint Controller card . . . . . . . . . . . . it

B.2 Joint \# 1 torque vs position . . . . . . . . . . . 19

B.3 Joint \# 2 torque vs position . . . . . . . . . . . 79

B.A Joint $\# 3$ torque vs position .................. so

B.5 Joint \# 4 torque vs position ................ s0

B.6 Joint \# 5 torque vs position ................. $\$$ s1

B.T Joint \# 6 torque vs position ................. s1

B.S Joint \# $i$ torque vs position .............. \$2

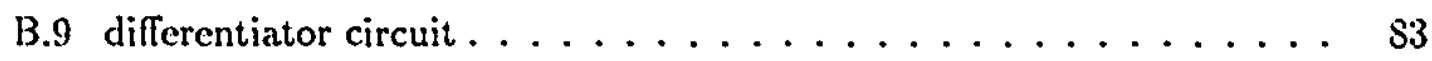

B.10 differentiator transfer function . . . . . . . . . . . $\$$ \$4 


\section{Chapter 1}

\section{Introduction}

The need for manipulators to perform various tasks has increased significantly in recent years and the tasks to be performed become more sophisticated. From simple pick and place operations, it is now intended to use robots in various applicalions such as power line maintenance, surgery, etc. These more delicate tasks repaire control systems which not only control the position of the robot, but, also permit, an interaction between the manipulator itself and the environment, on which it operates.

For a dynamic interaction during the task, pure position control is not sufliciem, an additional variable, the exerted force, must be kept under control. In fact, the regulation of the interaction between the force feedback and the desired position is required as opposed to tracking a reference position trajectory. $\Lambda n$ impedance controller will enable the modification of the mechanical impedance of a manipulator, in particular its apparent inertia, damping and stiffness. Such a controller adjusts the robot impedance and thus, the robot and the environment's dynamic interaction.

A decentralized impedance controller has been implemented on a high performance hydraulic manipulator: the Sarcos General Robotic Large Arm (GRLA). The Sarcos GRLA arm is a seven degree of freedom anthropomorphic manipulator.

This thesis is organized as follows. Following a brief literature review, a more 
elaborated definition of impedance control will be proposed and two types of implementation will be discussed. Next. the decentralized control algorithm will be ontlined. Theory, error characterization and simulation results are included. The next chapter presents the experimental results. The manipulator direct and inverse kinematics and hardware calibration are also discussed.

The decentralized impedance controller developed in this thesis permits a user to specify the Cartesian coordinates impedance of the robot as seen from the end-effector, but uses only the joint space variables, in contrast with other controllers forning the impedance loop in Cartesian coordinates. An analog controller is used in conjunction with a digital controller to compute the non-linear coupling cancellation between the joints. The proposed control law may be applied equally easily to ordinary or to redundant manipulators.

The cxperimental work presented in this thesis has been carried out at the Institut de recherche d'Hydro-Québec (IREQ), Robotics Division. Hydro-Québec is seriously involved in the development of robotics and telerobotics systems. The use of robots is presently considered for live power line maintenance, dam inspection, turbine maintenance and other tasks. Personel safety and productivity gain are the two main considerations. 


\section{Chapter 2}

\section{Literature Review}

\subsection{Introduction}

In general, the control of a robot may fall into three cases [33]: the control of position and orientation of a manipulator in a pre-determined reference frame; the control of a manipulator such that it will exert the specified forces and moments on its environment; and finally, the control of the position and orientation of a manipulator, taking into account the forces and moments interactions applied to the environment.

In this chapter, we will present a brief overvicw of the state of the art in robot. compliance control, in particular hybrid control and impedance control. These two control approaches not only take into account the position of the robot but also its interactions with the environment.

We will first discuss the basic concepts of constrained and unconstrained tasks and then the fundamentals of hybrid control and impedance control. We will then present the basic idea of decentralized impedance control. 


\subsection{Constrained and Unconstrained Tasks}

The control of a manipulator is related to the interaction between the control variables $(X, \dot{X}, \vec{X}, F)$ and the environment where it operates. We divide robot applications into roughly two different types of tasks: constrained and unconstrained tasks [32].

\subsubsection{Unconstrained tasks}

These tasks are characterized by negligible interaction forces between the robot and the cnvironment (such that, $d W=F \cdot d X \approx 0$ ) and for control purposes the manipulator can be considered as an isolated system. Its position. velocity and acceleration are the only controllable variables. In such situations, we can say that the manipulator operates in an unconstrained environment and this is when pure position control should be applied.

\subsubsection{Constrained tasks}

Constrained tasks occur when the robot position, velocity and acceleration are the negligible interaction variables. The controllable variable is now the interaction force between the robot and the environment. In this case, the robot is kinematically coupled with the environment. We need to further distinguish whether there is dynamic interaction between the manipulator and the environment.

If the force components are orthogonal to the displacement or if there is no displacement at all (case of a very stiff surface) then no dynamic interaction occurs and the mechanical work exchanged is approximately zero. In this special case, the correct control strategy is pure force control and since no dynamic interaction exists, the whole system (robot and environment) can still be considered as an isolated system (i.c. no exchange of energy).

The more general case occurs when the dynamic interaction between the robot 
and the environment is not negligible $\left(d H^{\circ}=F \cdot d X^{\circ} \neq 0\right)$. In this case, neither pure position control nor pure force control can be used successfully to control a robut because it is no longer an isolated system and the relationship between the control variables may change continuously. We must, consider a more general control law that. will take into account position. velocity, acceleration and also force as state variables.

- The command and control of a vector such as position or forct is not enough to control dynamic intcraction betueen systems. The controller must also command a relation between the port variables "iloxan

\subsection{Hybrid control}

The hybrid position/force control scheme was first proposed by Craig and Raibert [4] and almost ten years after, An and Hollcrbach [1] found that hybrid position/force control could go unstable. Also Zhang [34] found that it could go unstable due to kinematic conditions. Fisher and Mujtaba [12] found the source of the problem which was due to an incorrect formulation and implementation of the hybrid control scheme.

The main idea behind hybrid control is to separate the position and force constraints and independently analyze those control variables using pure force and pure position control laws along the proper coordinates. It is based on a decomposition of the task space. Given a task frame, a selection matrix (S) designates which axis will be position controlled and which will be force controlled. Once the joint torques from each part are computed, they are added to form the joint torque command to a simple set of actuators.

The Cartesian coordinates specifications $(x, f)$ are first mapped into the joint space specifications $(\theta, \tau)$. This mapping is computationally costly and problems will occur when the robot is in the vicinity of singular configurations because this mapping breaks down. Assuming small displacement, figure 2.1 presents the general hybrid 
control scheme.

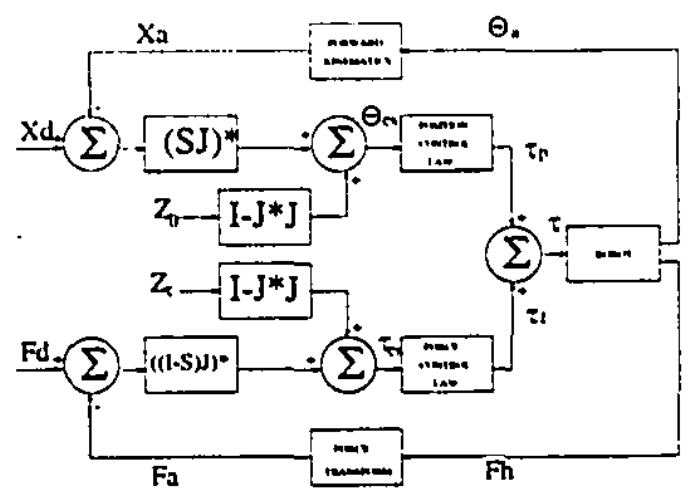

Figure 2.1: General hybrid control scheme

$X_{d}:$ desired position

$F_{d}:$ desired force

$S$ : Seleciıon matrix

$Z_{\theta}:$ arbitrary position vector in joint space

$Z_{\tau}:$ arbitrary torque vector in joint space

$0_{a}:$ joint set of actual position of robot

$F_{h}$ : force read by a force sensor

$\because(\cdot)^{*}=(\cdot)^{T}\left((\cdot)(\cdot)^{T}\right)^{-1}$

The force transform maps the sensed force frame into the task frame.

A position error $\left(X_{c}=X_{d}-X_{a}\right)$ and a force error $\left(F_{c}=F_{d}-F_{a}\right)$ are computed and mapped into joint space through a selection matrix to produce the angle and torque errors (the s subscript indicates that the selection matrix has been considered). Pure position control and pure force control are calculated based on the error vectors $\theta_{c s}$ and $\tau_{e s}$ respectively and each of them produce a desired torque. Those torques are added to form the torque that will drive the robot actuators. 


\subsubsection{Discussion}

Hybrid position/force control offers more possibilities than pure position or force control, but it has several drawbacks. For good performance. the drmamic model of the manipulator should be known since this control schene computes a torepue command to drive the robot actuators. It is consequently sensitive to the precision of the dynamic model of the robot. There is a second important problem. Assuming that. a good dynamic model is available, the environment of the robot must also be well known and defined. In certain cases, the choice of the control law for a particular degree of freedom may not be obvious. This control scheme applies to situations where the (known) environment is either stiff or soft. When a given environment is not compietely stiff nor completely soft, the robot cannot be controlled adeciuately. Another problem is due to the need to switch the selection matrix as the robot moves. Finally, Duffy [\$] found that the use of the selection matrix leads to dimensional inconsistency, and the results depend on the choice of units and on the choice of the origin of coordinates.

Despite its restrictions, hybrid position/force control is a step forward for dealing with constrained environments. In the next section, we discuss impedance control as a more general control approach.

\subsection{Impedance control}

Impedance control is concerned with the relationship between the position and contact force simultaneously and a single control law will be applied to both constrained and unconstrained tasks as opposed to hybrid control where two different control laws were necessary [18]. When there is no forces at the end-effector, the control reduces to a standard trajectory following problem in the task space. The control strategy does not need to be changed when contact is made between the cnvironment and 
the manipulator. The same controller is used in a single task including position and position/force subtasks. In this sense. impedance control leads to a more robust controller than the hybrid position/force controller since no switching is involved [6].

Impedance control is a gencral approach in which the robot is made to behave like a mass-spring-damper system whose parameters (J. inertia: B. damping; K. stiffness) can be specified arbitrarily in the task space. As opposed to pure force or pure position control, no attempt is made to track force or motion trajectories precisely but rather the relationship between force and motion is regulated. By tracking it is meant that the manipulator will be controlled to follow precisely a desired trajectory; by regulation it is meant that a desired trajectory will be given but the force exerted by the environment will not exceed a certain value corresponding to a specified impedance.

An impedance relates a flow to an effort $\left(Z=\frac{V}{l} \approx \frac{F}{V}\right)$. For maximum energy transfer, i.e. destruction of the environment in extreme cases, the robot's impedance must match the environment's impedance. For minimum energy transfer, i.e. delicate work, maximum mismatch is needed. The control establishes a dynamic relation between the position and force instead of just controlling one of these variables at any one time. Because of this dynamic interaction, the control scheme allows the manipulator to work in both constrained and unconstrained environment, and the environment itself can change during the task.

See figure $2.2^{1}$ for a general impedance control scheme. This scheme can be implemented either as position-based impedance control or as torque-based impedance control. Those two methods have different properties and the two approaches will be discussed later on.

Impedance control causes the robot to follow a desired trajectory as well as to

\footnotetext{
${ }^{1}$ from Whitney [32]
} 


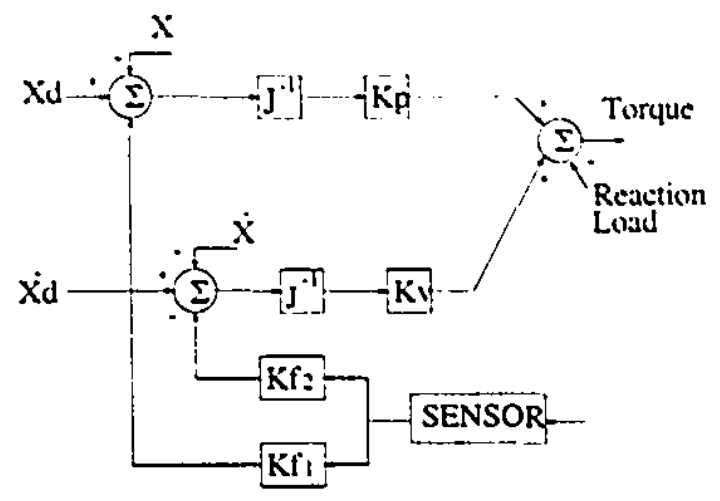

Figure 2.2: General impedance control scheme

exert a desired force when errors build up between the actual position and the desired position. The impedance controller will generate a force to correct the rolsol. trajectory. This correcting force catises the robot to follow a desired trajectory in free space. In constrained motion, this correcting force corresponds to be the interacting force between the robot and the task environment.

With impedance control, the contact force depends on the motion error of the robot end-effector, i.e. position, velocity and acceleration crrors [1S]. Refering to figure $2.3, m_{r}, b_{r}, k_{r}$ represent respectively the desired inertia, damping and stiffness of the robot. Also, $m_{e}, b_{e}, k_{e}$ are the environment impedance parameters. The basic dynamic equation that we wish the robot to follow is,

$$
m_{r}\left(\bar{x}-\ddot{x}_{d}\right)+b_{r}\left(\dot{x}-\dot{x}_{d}\right)+k_{r}\left(x-x_{d}\right)=\int_{e}
$$

where

$$
f_{e}=m_{e} \ddot{x}+b_{e} \dot{x}+k_{e} x
$$

The variables $f_{e}$ and $x$ are respectively the environment contact force and the actual position of the robot. The variable $x_{d}$ represents the nominal trajectory and we can relate it to the equivalent force input to the system $\left(f_{\text {in }}\right)$. In fact, $\int_{\text {in }}$ represents the force that should be exerted by the robot in order to follow the desired trajectory. 


$$
m_{\mathrm{r}} \ddot{x}_{d}+b_{r} \dot{x}_{d}+k_{r} x_{d} \equiv f_{i n}
$$

Equation 2.3 represents what would be the response if the robot was operating in free space. But if the robot encounters a wall of a given impedance $z$ then $f_{\text {in }}$ is compared to the sensed force to produce a modified trajectory using equation 2.4 that takes care of the interaction of the robot with the environment. Finally, we say that the overall behavior of the coupled robot/environment system is ideally given by equation 2.4 .

$$
\frac{x}{f_{\text {in }}}=\frac{1}{\left(m_{r}+m_{c}\right) s^{2}+\left(b_{r}+b_{c}\right) s+\left(k_{r}+k_{c}\right)}
$$

In stiffncss control and damping control, the contact force is treated as arising from the position and the velocity errors respectively: In impedance control, the contact force depends on the motion error of the robot end-effector, i.e. position, velocity and acceleration errors [1S]. Stiffness control is a special case of impedance control where only the steady state force-displacement relationship is considered $\left(x=\frac{f_{w+1}}{\left(k_{r}+k_{e}\right)}\right)$; damping control is a special case of impedance control where only the force-velocity relationship is considered $\left(\dot{x}=\frac{f_{i n}}{\left(b_{r}+b_{e}\right)}\right)$.

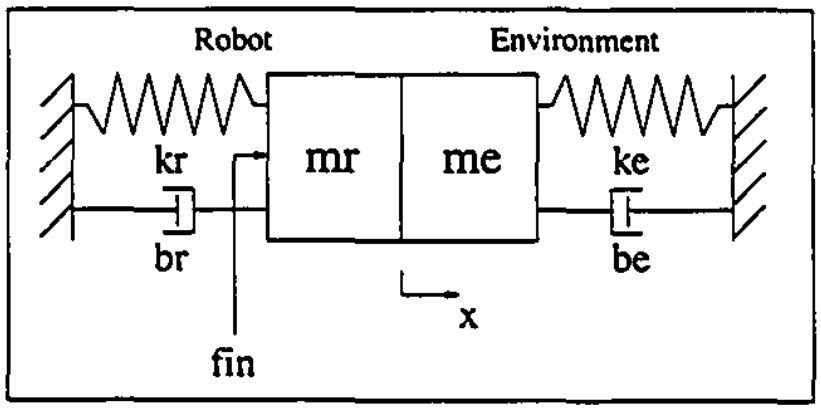

Figure 2.3: Robot and environment system modeled with linear impedances

A parallel with pure position control and pure force control can also be made. Pure position control corresponds to the case of an infinite impedance (or $Z_{r} \gg Z_{\mathrm{e}}$ ) 
and pure force control to the case of a zero impedance (or $Z_{r} \ll \%_{r}$ ).

No problems are caused by the switching of the selection matrix as it is the atse with hybrid control, since the impedance itself manages the change by a dynamic interaction with the enviromment. Also. there is no need to know exactly the impedance (or rather the admittance [1 7$]$ ) of the environment to acheive a good performance ( $r e$ atively to hybrid position/force control). With the same control algorithm a robot can be controlled in free space and in constrained enviromments because the impedance specifies the dynamic relation between force and position. This is not the case with hybrid control: in case of a rapid change in the environment. stability is not guaranteed (e.g. a pure force controller cannot be used in free space since the robot would continually accelerate; a similar problem arises if pure position control is used in a constrained environment, the force applied by the robot would be theoretically infinite).

We now present two different approaches to implement impedance control. Both are based on the same concept but the performance can vary depending on the available hardware, and each method has its own advantages and disadvantages. If impedance control is to be implemented with robots that were not designed for trading force, there will be little choice for the implementation, but in other cases at choice may be possible.

Also, one should keep in mind that in practical implementations, the acceleration term that could contribute to change the robot inertia, is difficult to obtain. Differentiating twice the position signal increases the noise component of the signal, leading to useless information. Filtering the position signal prior to differentiating is one approach. However, during rapid changes in acceleration, filtering will degrade the shape of the signal. A possible approach is to estimatc accelcration with state space methods but it is not clear whether this is feasible, or even worth the trouble. 


\subsubsection{Position-based impedance control}

The position-based impedance control approach depends on an accurate internal position control loop around which is built the impedance control loop. The forces are sensed and position commands are used as an input for the internal position loop. Since many industrial robots are provided with accurate position controllers, this approach is a simple way to enhance the performance of the system by using retro-fitted force feedback.

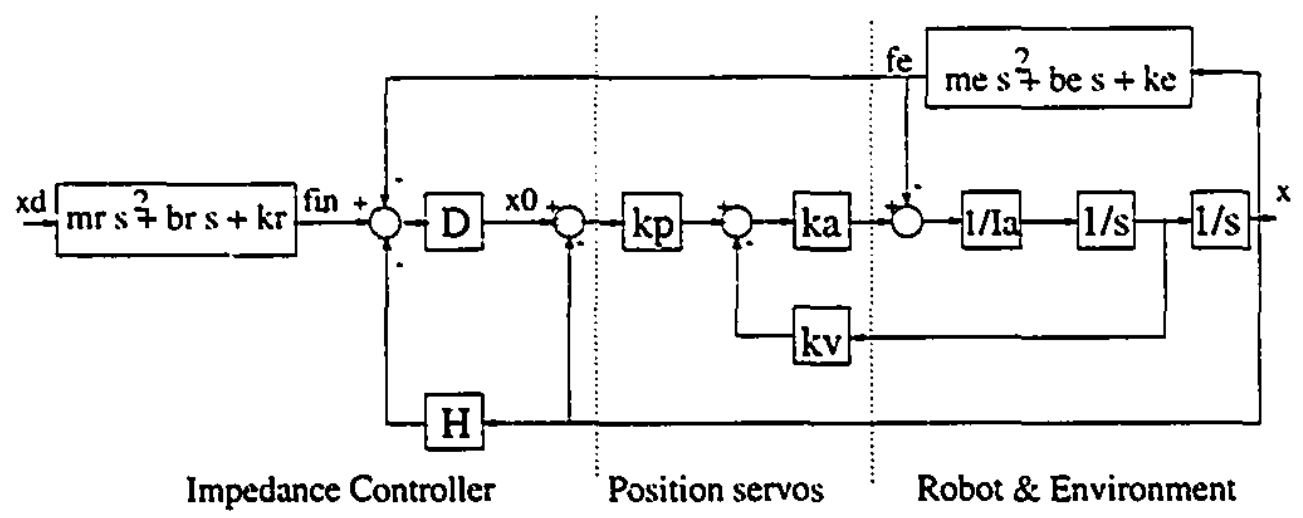

Figure 2.4: Position-based impedance control scheme

The main advantage of this method is that the dynamic model of the robot is not required and rather good performance can be demonstrated. Developing a dynamic model can be laborious and will require costly computations for its calculation. This will affect the stability and the robustness of the system. The inner servo loop gains (PD gains) must be set as high as possible. In this situation, the robot dynamics is seen like a disturbance to the impedance controller and are rejected by the position scrvo loop (see figure 2.4). The terms $\mathbf{D}$ and $\mathbf{H}$ may vary depending on the implementation [25]. It is important that the position servo loop has a much shorter time constant than the impedance loop, because the internal servo loop tracks a desired position.

One problem with the position-based impedance control method is the difficulty 
to select a soft impedance without encountering stability problems [2:3]. This is due to the fact that position is uscd as a command to the position leop. This position command is the result of $\frac{f_{n}(s)-f_{-}(s)}{Z_{r}(x)}$. In the steady state case. for cxample, at smatl stiffness produces a large position command and may cxcite high frequency resonant modes to produce instability.

The transfer function derived from a position-based implementation (with $\mathbf{H}=0$ ) and $\mathrm{D}=\frac{1}{m_{r} s^{2}+b_{r} s+k_{r}}$ ) resembles the ideal one (equation 2.4) but contaius an crror term that is minimized with high PD controller gain $\left(k_{i}\right.$, and $\left.k_{p}\right)$.

$$
\frac{x}{f_{\text {in }}}=\frac{1}{\left(m_{r} s^{2}+b_{r} s+k_{r}\right)+\left(m_{r} s^{2}+b_{r} s+k_{r}\right)+\Delta(s)}
$$

where

$$
\Delta(s)=\frac{\left(m_{r} s^{2}+b_{r} s+k_{r}\right)\left(\left(I_{a}+m_{r}\right) s^{2}+b_{r} s+k_{r}\right)}{k_{w} s+k_{p}}
$$

There is a steady state error with respect to the ideal impedance control equation (equation 2.4). If $k_{v}$ and $k_{p}$ are large enough as compared to the desired impedance, then $\Delta(s)$ can be neglected and (2.5) is similar to the ideal transfer function (2..1). If $k_{v}$ and $k_{p}$ are not sufficiently high then the manipulator dynamics (the inertia in our case) and the environment affect the servo loop and degrade the performance (note that the environment must be taken into account in the impedance loop but not in the servo loop).

There exists various implementations of the position based method, see [2:5] [1:3] [18] for example. In [25], it is shown that the error on the steady state performance may be eliminated in cases where the PD gains cannot be set sufficiently high. 


\subsubsection{Torque-based impedance control}

In general, the torque based approach to impedance control, figure $2.5^{2}$, will theorctically lead to more efficient and precis. impedance controllers. The major difference ats compared to the position based approach is that it requires an accurate dynamic model of the robot manipulator. In fact, the principle is to invert the dynamics of the system and then to set the desired impedance.

In this control scheme, the actual positions, velocities and forces are sensed and are then compared to their respective state variables. The error between the desired and actual state variables are combined with the desired Cartesian coordinates impedance to produce a desired torque command to the actuators.

Since this approach directly controls the torque command to the robot's actuators, the stability and robustness of the overall system depends on the precision of the dynamic model. In fact, it can be stated that in practice, this method has poor disturbance rejection because no inner loop is used inside the impedance loop to compensate for parametric uncertainties.

Figure 2.6 shows a qualitative relationship between the effect of parameter uncertaintics and the error of the torque-based controller. Of course when the parametric uncertainties are small, torque based methods offer a better performance but the value of $\alpha$ is small. Above $\alpha$, torque based controllers rapidly loose their performances and become unstable [2].

The problem with the dynamic model is twofold. First, there always exist modelling errors since it is impossible to completely model any system. Thus, the controller must to some degree be robust to parameter uncertainty. Second, the calculations required for the inverse dynamic's model increase rapidly with the complexity of the model and may lead to difficulties in real time computing. As computers become faster. the importance of this problem will be reduced but will always linger.

\footnotetext{
"from kihatib [21]
} 
As opposed to the position based method. stability is not affected when soft impedances are required but it may be diffeult to produce very stiff imperlances. In the position based method, a position command was produced from the ratio of a force over the desired impedance. In the torque based method. a torque command is produced from the product of a position and a desired impedance. In the case of a large impedance, the bandwidth of the torque command increases and may again excite unmodelled dynamics.

In summary, the more perfectly the plant is known, the better the controller performance will be. In the torque based method, a dynamic model of the robot. is mandatory but it will offer better performance. More time will be needed in the modelling part as compared to the position-based method where almost, no modelling is required.

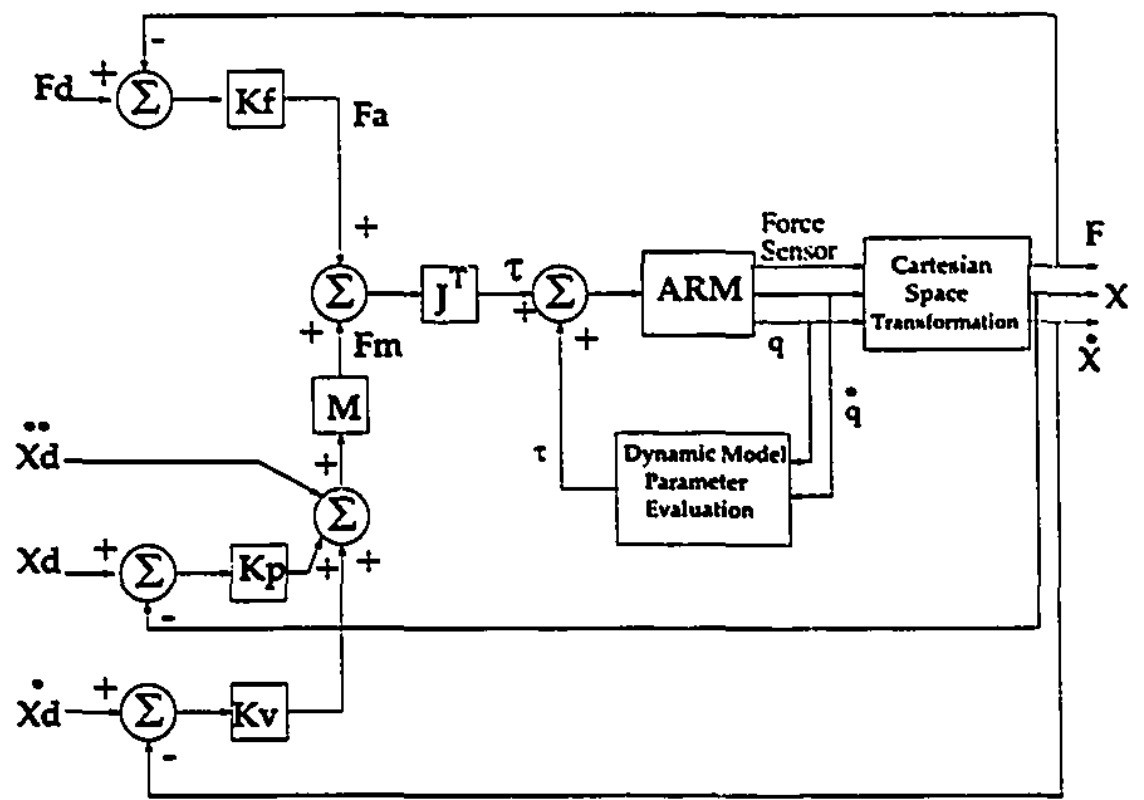

Figure 2.5: Torque-based impedance control 


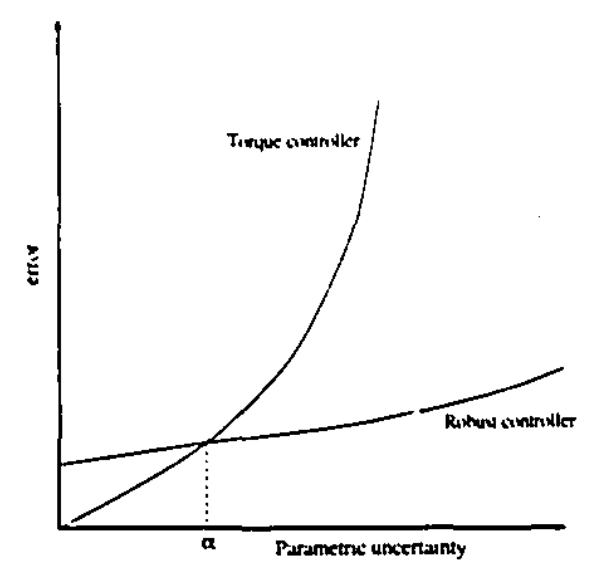

Figure 2.6: Parametric uncertainty

\subsection{Decentralized control}

Deriving inspiration from the consideration of human stiffness control by skeletal muscles, the end-effector compliance of a robot can be specified by directly altering its joint stiffness [20]. This is the basic concept of the proposed method and it will be extended to damping and inertia control to specify in Cartesian coordinates the impedance of a manipulator from the joint space variables.

Salisbury [2T] pioneered active stiffness control based on a joint torque control law which can be used to acheive any desired stiffness of the end-effector in the compliance frame. An error on the stiffness would appear as the manipulator is deflected far from its nominal position leading to a curved path in space rather than a straight line motion [24]. A fundamental advantage is owed to the fact that the inversion of the Jacobian matrix is not needed, but only its transpose. Kaneko et al. [20] proposed a direct compliance control method inspired from Salibury's and is based on a completely independent joint control, leading to any desired compliance, and simplifying the complexity of the control law. An important drawback arises from the impiementation. In order to be able to control in Cartesian coordinates (three position and three orientations), a 21 DOF robot would required. Also, the method 
has another problem. the end point compliance camot be arbit rarily set according to the arm configuration. This is due to the negative joint compliance required in certain arm configurations [19] [31].

The interesting point however is that simple SISO controllers can be used in conjunction with co-located sensors and actuators.

\subsection{Other methods}

The position, force and compliance control of robots has received a lot of interest. from the research community and many other methods have been proposed. Most. of them are based on torque control and present different approaches and methods to take into account parametric uncertainties of the dynamic model. Among the proposed solutions we find adaptive impedance controllers [28], sliding mode control [35], etc. Those implementations appear to offer good performances, but are usually computationally costly and are not necessarily casy to implement in practice. Since the focus of this thesis is on simple and experimentally provable approaches, these will not be discussed further. 


\section{Chapter 3}

\section{Decentralized control of impedance}

\subsection{Basics}

Joint space controllers are used to control the impedance in Cartesian coordinates. The implementation proposed here requires joint torque sensors located in the joints of the manipulator. The position and force feedback control signals are based only on joint variables: torque and displacement. The fundamental advantage of this controller is that it does not require any coordinate transformation in the inner loop, only linear SISO compensators are needed. Co-located torque sensors lead to a larger control bandwidth since the structural dynamics of the arm is seen as a perturbation, but is not part of the plant to be controlled. Simple SISO controllers designed to modulate individual joint impedances can achieve diagonal (decoupled) impedance matrices in Cartesian coordinates, provided that another set of SISO compensators, called here "decouplers", are set up to cancel the couplings among the joints. This strategy results in a simple computational architecture which does not require complex coordinate transformations to be performed at servo rate. 
Given a desired inmedance in (artesian coordinates, it is mapped into joint coordinates. Assuming small displacements. we linearize to obtain:

$$
\begin{aligned}
\tau & =J^{T} F \\
\delta X & \approx J \delta 0
\end{aligned}
$$

then, in the Laplace domain,

$$
\begin{aligned}
& F(s)=Z_{c}(s) \theta(s) \\
& \tau(s)=Z_{\theta}(s) \theta(s)
\end{aligned}
$$

thus

$$
Z_{\theta}(s)=J^{T} Z_{c}(s) J
$$

where $Z_{c}(s)=B_{x} s+K_{x}$

For stiffness alone, the matrix $Z_{\theta}$ has been characterized and called by Salisbury the joint stiffness matrix. There we look at a more general case where impedance can be arbitrary. No Jacobian inversion is required, reducing the computations and alleviating problems near kinematics singularities. There is however no reason why the resulting $Z_{\theta}(s)$ should be diagonal (and not necessarily positive definite) when $Z_{c}(s)$ is chosen to be diagonal, except in special cases.

Conversely, a diagonal $Z_{\theta}(s)$ (namely PD control) will not lead to diagonal $Z_{c}(s)$ : in this case, cross coupling occurs in Cartesian space. When $Z_{c}(s)$ is diagonal, $Z_{0}(s)$ is symmetric and the terms outside the diagonal (cross coupling terms) are not negligible. However, in general the diagonality or degree of decoupling of $Z_{0}(s)$ will vary with the robot configuration. The significance of a non diagonal $\%_{0}(s)$ is that the errors in one joint will affect the commanded torque in all the other joints as was pointed out in [27](see equation 3.4). Only certain cases will lead to decoupling which is an architectural kinematic property of the underlying mechanism as shown in [16]. 
In certain situations, redundancy can contribute to reduce or even cancel cross coupling terms by exploiting the additional degree(s) of freedom to select a configuration which minimizes the off diagonal terms of the joint space impedance matrix for a given Cartesian coordinates impedance matrix. The general relation between the number of parameters to control in joint space and the number of degrees of freedom is given by:

$$
p=\sum_{i=0}^{N-1}(-1)^{i}(N-i)^{2}
$$

where $\mathrm{N}$ is the number of D.O.F. and p, the number of parameters to control.

For purposes of illustration, the case of a planar robot with three DOF is now discussed. It is possible to configure a manipulator of this kind such that, for a given diagonal Cartesian impedance matrix, the joint space impedance matrix becomes diagonal. This can be explained by the fact that the Cartesian impedance matrix specifies three terms, the impedance in the $x$ and $y$ directions, the cross coupling term between $x$ and $y$ being fixed to zero, ideally. Since the robot has three joints it can be verified that there is a unique solution to three equations with three unknowns. Redundancy can be used to yield a diagonal joint space matrix that will satisfy the Cartesian impedance matrix.

In the more general case of a seven degree of freedom robot, from equation 3.6, we find that 28 parameters are to be determined, among which 21 must be set to zcro. In this case, a manipulator with at least $2 S$ degrees of freedom would be capable of yiclding a diagonal Cartesian coordinates impedance matrix from a diagonal joint impedance matrix. Moderate redundancy, in this case, can only contribute to minimize the cross coupling terms. An optimization method could be applied to find postures that minimize the coupling but decoupling cannot occur in general.

To set the diagonal terms of $Z_{\theta}(s)$ by means of feedback control, the gains of the joint PD controllers are scheduled or continuously modified as the robot moves. Since 
the off diagonal terms are neglected. the system will be highly coupled hat the control is quite robust. From equation 3.4. for the stiffness term (and similarly for a genteral impedance), the torque command to one joint is given by:

$$
\begin{aligned}
& \tau_{i}=\sum_{j=1}^{N}\left(\Lambda_{i, j} \theta_{j}\right) \\
& \tau_{i}=\kappa_{i, i} \theta_{i}+\sum_{j=i, j \neq i}^{N} \kappa_{i, j} \theta_{j}
\end{aligned}
$$

Equation 3.7 separates into two parts as in equation 3.S. 'The diagonal terms are readily obtained with PD controllers about each joint. The remaining terms correspond to compensating torques calculated from the off diagonal terms and from the position of other joints. The resulting impedance matrix in Cartesian coordinates is now exactly decoupled using joint measurements alone.

One advantage of this method, is that analog PD controllers may be used to implement the diagonal terms of the joint space stiffnesses and clamping, thereby freeing the system from sampling and digitization approximations. The cross coupling terms are computed numerically and summed with the PD controller output torgute to yield the actuator force demand signal. An additional computed toreue may be: used to fine tune the system.

\subsection{Transfer function}

Decentralized impedance control operates in joint space. It is nevertheless importint. to derive the Cartesian coordinates transfer function. It will be obtained by mapping the joint coordinates control into a Cartesian coordinates. The dynamics: incrtia, damping and stiffness are captured by, 


$$
\tau_{\text {rotat }}=D_{\theta} \ddot{\theta}+V(\theta, \dot{\theta})+C^{\prime}(\theta)
$$

applying the control,

$$
\begin{aligned}
\tau_{\text {robot }} & =M_{\theta}\left(\vec{\theta}_{d}-\ddot{\theta}\right)+B_{\theta}\left(\dot{\theta}_{d}-\dot{\theta}\right)+\kappa_{\theta}\left(\theta_{d}-\theta\right) \\
& +\dot{V}(\theta, \dot{\theta})+\dot{G}(\theta)
\end{aligned}
$$

results in,

$$
\begin{aligned}
D_{\theta} \ddot{\theta}+V(\theta, \dot{\theta})+G(\theta) & =M_{\theta}\left(\ddot{\theta}_{d}-\dot{\theta}\right)+B_{\theta}\left(\dot{\theta}_{d}-\dot{\theta}\right)+K_{\theta}\left(\theta_{d}-\theta\right) \\
& +\dot{V}(\theta, \dot{\theta})+\hat{G}(\theta)
\end{aligned}
$$

assuming,

$$
\begin{aligned}
\tau_{d} & =M_{\theta} \ddot{\theta}_{d}+B_{\theta} \dot{\theta}_{d}+K_{\theta} \theta_{d} \\
\tau & =J^{T} F \\
\delta x & \approx J \delta \theta \\
\ddot{x} & =J \ddot{\theta}+j \dot{\theta}
\end{aligned}
$$

we have,

$$
\begin{aligned}
F_{d} & =J^{-T}\left(M_{\theta}+D_{\theta}\right) \ddot{\theta}+J^{-T} B_{\theta} \dot{\theta}+J^{-T} K_{\theta} \theta+J^{-T}(V-\hat{V}) \\
& +J^{-T}(G-\dot{G}) \\
F_{d} & =J^{-T}\left(M_{\theta}+D_{\theta}\right) J^{-1} \ddot{x}+J^{-T} B_{\theta} J^{-1} \dot{x}+J^{-T} K_{\theta} J^{-1} x+ \\
& +J^{-T}(V-\hat{V})+J^{-T}(G-\hat{G})-J^{-T}\left(M_{\theta}+D_{\theta}\right) J^{-1} \dot{j} \dot{\theta}
\end{aligned}
$$

where

$$
\begin{aligned}
M_{\theta} & =J^{T} M_{x} J \\
B_{\theta} & =J^{T} B_{x} J \\
K_{\theta} & =J^{T} K_{x} J \\
\mathcal{D} & =J^{-T} D_{\theta} J^{-1}
\end{aligned}
$$


using the above cquations we have.

$$
\begin{aligned}
& F_{d}=\left(\left(\mathcal{D}+M_{r}\right) s^{2}+B_{s} s+K_{x}\right) \cdot r+\Delta_{r r r u r} \\
& \Delta_{\text {error }}=J^{-T}(V-\hat{l})+J^{-T}(C-\hat{C})-J^{-T}\left(M_{0}+D_{0}\right) \cdot J^{-1} . j i
\end{aligned}
$$

When a relatively low speed of motion is reguired. the centrilugal and coriolis term become negligible and also does the rate of change of the Jacobian matrix. The error inherent to the gravity term will be discussed later and for now we will assume perfect compensation. The transfer function thus becomes.

$$
\frac{x}{F_{d}}=\left(\left(\mathcal{D}+M_{x}\right) s^{2}+B_{s} s+K_{r}\right)^{-1}
$$

By abuse of notation, $F_{d}$ means forces and torques and by $x$, position and orientation. 'The ratio $\frac{x}{F_{d}}$ is used to represent the transfer functions in cach direction (i.e. $\frac{x_{x}}{F_{d_{x}}}, \frac{x_{x}}{F_{d_{y}}}, \frac{x_{x}}{F_{d_{z}}}, \frac{x_{y}}{F_{d_{x}}}, \frac{x_{y}}{F_{d_{y}}}, \frac{x_{y}}{F_{d_{z}}}, \frac{x_{z}}{F_{d_{x}}}, \frac{x_{z}}{F_{d_{y}}}, \frac{x_{*}}{F_{d_{z}}}$ ). Notice that the robot inertia appears in equation 3.24. Since this matrix is not diagonal it will cause errors in the transient. response. These errors can be minimized using the redundancy of the manipulator when it is available. The error characterization will be discussed in section 3. $\pi$.

From equation 3.24, we find that the steady state value is,

$$
\lim _{s \rightarrow 0} \frac{\Delta x}{\Delta F_{x}}=K_{x}^{-1}=\left(\begin{array}{ccc}
\frac{1}{k_{x}} & 0 & 0 \\
0 & \frac{1}{k_{y}} & 0 \\
0 & 0 & \frac{1}{k_{z}}
\end{array}\right)
$$

\footnotetext{
'Properly speaking, the above equations would apply to rotations only if they are sinall. In section 3.7 a correction factor is derived to correct this problem.
} 


\subsection{Continuous Time Stability}

Many non-linear feedback laws have been proposed in the literature for the control design of robotic systems modelled by rigid body equations of open-kinematic chains. Predominant among these techniques is the computed torque approach. This approach has, in practice, a number of drawbacks because the global stability depends on exact dynamic models and full knowledge of the parameters of the system. Accuracy and speed in computation is also required [22]. In this section. we will show that the proposed controller remains stable. In order to prove the stability, we will use the state space theory. Assuming negligible coriolis and centrifugal terms and using gravity compensation we have:

$$
D_{\theta} \ddot{\theta}=B_{\theta}\left(\dot{\theta}_{d}-\dot{\theta}\right)+\kappa_{\theta}\left(\theta_{d}-\theta\right)
$$

forming a desired torque,

$$
\tau_{d}=B_{\theta} \dot{\theta}_{d}+K_{\theta} \theta_{d}
$$

we have

$$
\tau_{d}=D_{\theta} \ddot{\theta}+B_{\theta} \dot{\theta}+K_{\theta} \theta
$$

Then, with $x$ a state variable (not a Cartesian coordinates vector),

$$
\begin{aligned}
& \dot{x}=A x+B u \\
& y=C x
\end{aligned}
$$


where

$$
\begin{aligned}
x & =\left(\begin{array}{l}
\theta \\
\dot{\theta}
\end{array}\right) \\
A & =\left(\begin{array}{cc}
0 & I \\
-D_{\theta}^{-1} K_{\theta} & -D_{\theta}^{-1} B_{\theta}
\end{array}\right) \\
B & =\left(\begin{array}{c}
0 \\
D_{0}^{-1}
\end{array}\right) \\
C & =\left(\begin{array}{ll}
I & 0
\end{array}\right) \\
u & =\tau_{d}
\end{aligned}
$$

The characteristic equation, $\Delta(s)$, is given by the determinant of $(s /-. l)$ :

$$
\begin{aligned}
(s I-A) & =\left(\begin{array}{cc}
s I & -I \\
D_{\theta}^{-1} K_{\theta} & s I+D_{\theta}^{-1} B_{\theta}
\end{array}\right) \\
\Delta(s) & =D_{\theta}^{-1}\left(D_{\theta} s^{2}+B_{\theta} s+K_{\theta}\right)=0
\end{aligned}
$$

The system will be stable if the given impedance transfer function in Carlesian coordinates is stable. The characteristic equation (equation 3.38 ) will have all its roots in the left half plane if and only if:

1. $D_{\theta}, B_{\theta}$ and $K_{\theta}$ are positive semidefinite

2. $D_{0}$ or $K_{\theta}$ is positive definite [5]

The inertia matrix $D_{\theta}$ has the property of being always positive definite simply because the quadratic $\ddot{\theta}^{T} D_{\theta} \vec{\theta}$ represents the work effected to overcome the joint inertias and therefore must be greater than zero [34]. If an acceleration feedlack is to be used, we simply need to make sure that $\left(D_{0}+M_{0}\right)$ is positive definitc. Also $B_{0}$ and $K_{\theta}$ are positive semidefinite because of thcir structural propertics,

$$
\begin{aligned}
& K_{\theta}=J^{T} K_{x} J \\
& B_{\theta}=J^{T} B_{x} J
\end{aligned}
$$


then,

$$
\begin{aligned}
K_{\theta} & =J^{T} h_{r x}^{T} K_{r x} J \\
B_{\theta} & =J^{T} B_{r x}^{T} B_{r x} J \\
r_{r x} & =\sqrt{\cdot} \\
K_{\theta} & =Q^{T} Q \\
Q & =\cdot_{r x} J
\end{aligned}
$$

From the above, we can conclude that $B_{\theta}$ and $K_{\theta}$ are positive definite if $Q$ is not singular and positive semidefinite if $Q$ is singular.

It is known that a change in coordinates (e.g. from joint to Cartesian coordinates) will not affect the stability, thus the proposed control law will remain stable at the Cartesian level [15].

\subsection{Discrete Time System Stability}

The sampling frequency of a digital controller greatly affects the bounds within which such a system is stable. It is known that stable continuous time systems may become unstable when an equivalent digital controller is applied in replacement. In the set-up used in this thesis, some parts will be implemented using an analog controller (the diagonal terms of the damping and stiffness matrices) and the decoupler part will be implemented digitally. To simplify this section, we will study the system as if the controller was completely digital.

Digital controllers introduce a time delay and cause a reduction of the phase margin leading to instabilities. The analysis of linear SISO digital systems is relatively simple in the case of low order systems and may be easily generalized to muiti-variable systems when they are fully decoupled (diagonal matrices) since there is no interaction 
between the different inputs and outputs. Enfortunately, the case of coupled multivariable systems does not generalize as casily. We will first discuss the one dimension case.

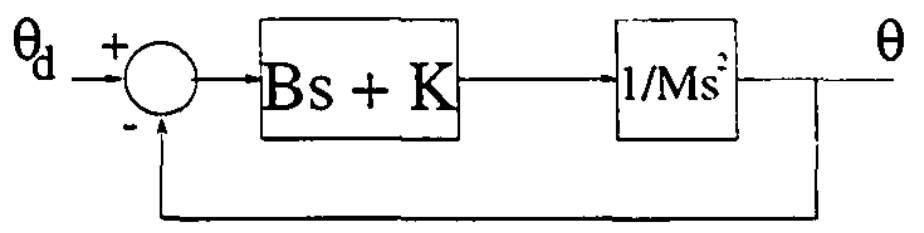

\section{Analog System}

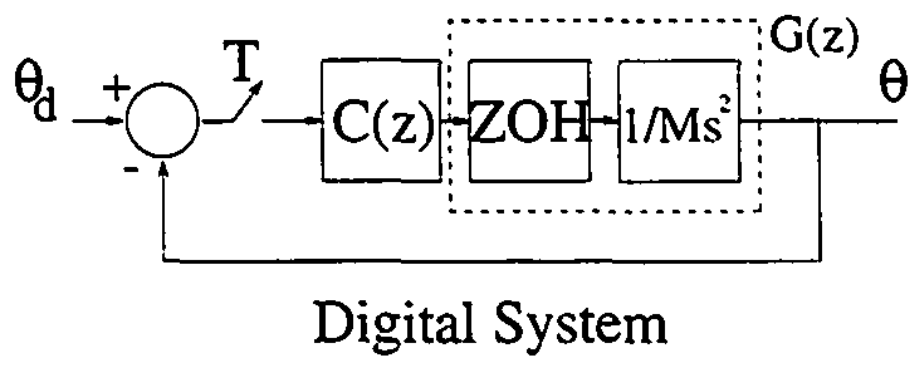

Figure 3.1: Analog and Digital Systems

Various approximations may be selected to analyze the system in figure 3.1 , a жro order hold equivalent has been chosen. An equivalent digital controller is used to al)proximate the continuous one. Using Tustin's method [14] (bilinear transformation), given a sampling period $\mathrm{T}$, we have in the $\mathcal{Z}$ domain,

$$
\begin{aligned}
s & =\left(\frac{2}{T} \frac{z-1}{z+1}\right) \\
C(z) & =B\left(\frac{2}{T} \frac{z-1}{z+1}\right)+K \\
G(z) & =\frac{T^{2}}{2 M} \frac{z+1}{(z-1)^{2}} \\
\text { open loop } & =\frac{\left(2 B T+K^{\prime} T^{2}\right) z+\left(K^{\prime} T^{2}-2 B^{\prime} T\right)}{2 M(z-1)^{2}} \\
\text { closed loop } & =\frac{C(z) G(z)}{1+C(z) G(z)}
\end{aligned}
$$




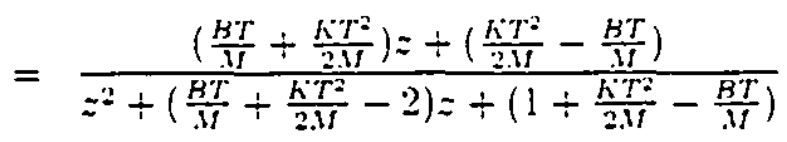

$\overrightarrow{\mathcal{E}}$ domain systems are stable when all their poles are included inside the unit circle. lising the Jury stability test ([1.1]) the following cuiditions for stability are foind:

$$
\begin{aligned}
& K<\frac{2 B}{T} \\
& B<\frac{2 M}{T} \\
& K>0
\end{aligned}
$$

The same conditions apply in the case of diagonal multi-tariable systems except that $K, B, M$ are diagonal matrices.

\subsubsection{Coupled multi variable case}

The complete study of coupled multi-variable systems may become relatively complex and will not be treated in this thesis. From simulation, it appears that the results obtained using the stability conditions for each elements of the matrices $M, B$ and $K$ produce limits in the same order that the effective stability limit.

With coupled systems, the response in one direction affects the response in an another direction and energy may be exchanged among the joints. Energy is not necessarily decreasing and the system may become non passive.

Given the continuous time system, we have from section 3.3,

$$
\begin{aligned}
& A=\left(\begin{array}{cc}
0_{N \times N} & I_{N_{x N}} \\
-D_{\theta}^{-1} K_{\theta} & -D_{\theta}^{-1} B_{\theta}
\end{array}\right) \\
& B=\left(\begin{array}{c}
0_{N \times N} \\
D_{\theta}^{-1}
\end{array}\right)
\end{aligned}
$$




$$
\begin{aligned}
& C=\left(\begin{array}{ll}
C x & 0 x \ldots
\end{array}\right) \\
& \dot{r}=A x+B u \\
& y^{\prime}=C r
\end{aligned}
$$

And the continuous time solution is given by:

$$
\begin{aligned}
& x(t)=c^{* t} x(0)+\int_{0}^{t} c^{A \tau} B u(\tau) d \tau \\
& y(t)=C x(t)
\end{aligned}
$$

The discrete time equivalent ( $\mathrm{T}$ being the sampling period) is.

$$
\begin{aligned}
x(n+1) & =e^{A T} x(n)+\int_{0}^{T} e^{A \tau} B u(\tau) d \tau \\
& =e^{A T} x(n)+\left(\int_{0}^{T} c^{A T} B d \tau\right) u(n) \\
y(n) & =C x(n)
\end{aligned}
$$

or in the $\mathbf{z}$ domain.

$$
\begin{aligned}
\left(z I-e^{A T}\right) X(z) & =\left(\int_{0}^{T} e^{A T} B\right) U(z) \\
X(z) & =\left(z I-c^{A T}\right)^{-1}\left(\int_{0}^{T} c^{A \tau} B\right) U(z)
\end{aligned}
$$

The digital system will be stable if all the roots of $\operatorname{Det}\left(z J-c^{A T}\right)$ lie inside the unit circle. If $\mathrm{T}$ is sufficiently small, an approximation for $e^{\lambda T}$ can be used:

$$
\begin{aligned}
e^{A T} & \approx I+A T \\
\left(z I-e^{A T}\right) & =\left(z I-I-e^{A T}\right) \\
\operatorname{Det}\left(z I-e^{A T}\right) & =\operatorname{Det}\left(\begin{array}{cc}
(z-1) I_{N 土 N} & -I_{N \pm N} T \\
D_{\theta}^{-1} K_{\theta} T & (z-1) I_{N 土 N}+D_{\theta}^{-1} B_{\theta} T
\end{array}\right)
\end{aligned}
$$


Finding the mathematical symbolic expression of the roots of the determinant in the previous equation is very difficult even in the 2 degrees of freedom case since it leads to a $1^{\text {th }}$ order equation. In the case of a seven degrees of frecdom robot, the equation is of the $14^{\text {th }}$ order. Applying the Jury stability test leads to complex calculations. The symbolic compıtation of the determinant is difficult but if numerical values are given for $D_{\theta}^{-1}, B_{\theta}$ and $K_{\theta}$ then it is easy to verify the location of the roots of the determinant of $\left(z I-c^{A T}\right)$.

$$
\begin{aligned}
\Delta(z) & =\operatorname{Det}\left(z I-\epsilon^{A T}\right) \\
& =\prod_{i=1}^{N}\left(z-z_{i}\right)
\end{aligned}
$$

The system is stable if

$$
z_{i}<|1|, \forall i
$$

It has also been shown that a change in coordinates should not affect the stability conditions of the system under study [15]. If the Cartesian coordinates inertia matrix is diagonal, it is easy to study the stability of the system since it is as simple as the one dimensional case. In the case of a redundant robot, a given position can be reached from many configurations and to each of these configurations corresponds a different inertia matrix. An optimization criterion can be used to select the best pose, reducing the coupling, and at the same time simplifying the analysis of the stability conditions.

\subsection{Controllability and Observability}

From equations (3.33), (3.34) and (3.35),

$$
A=\left(\begin{array}{cc}
0 & I \\
-D_{\theta}^{-1} K_{\theta} & -D_{\theta}^{-1} B_{\theta}
\end{array}\right)
$$




$$
\begin{aligned}
& B=\left(\begin{array}{c}
0 \\
D_{\theta}^{-1}
\end{array}\right) \\
& C=\left(\begin{array}{ll}
I & 0
\end{array}\right)
\end{aligned}
$$

The controllability and observability matrices are given by:

$$
\begin{aligned}
& \mathcal{C}=\left(\begin{array}{lllll}
B & A B & A^{2} B & \ldots & A^{n-1} B
\end{array}\right) \\
& \mathcal{O}=\left(\begin{array}{c}
C \\
C A \\
C A^{2} \\
\vdots \\
C A^{n-1}
\end{array}\right) \\
& C=\left(\begin{array}{ccc}
0 & D_{\theta}^{-1} & \ldots \\
D_{0}^{-1} & -D_{\theta}^{-1} B_{\theta} D_{\theta}^{-1} & \ldots
\end{array}\right) \\
& \mathcal{O}=\left(\begin{array}{cc}
I & 0 \\
0 & I \\
\vdots & \vdots
\end{array}\right)
\end{aligned}
$$

The system is controllable if $\operatorname{rank}(\mathcal{C})$ is $n$, when $A$ is $n \times n$. From equalion 3.78, we notice that a sufficient condition for $\mathcal{C}$ to have full rank is that the determinant of

$$
\mathcal{C}_{1}=\left(\begin{array}{cc}
0 & D_{\theta}^{-1} \\
D_{\theta}^{-1} & -D_{\theta}^{-1} B_{\theta} D_{\theta}^{-1}
\end{array}\right)
$$

is different from zero. Since the inertia matrix, $D_{0}$, is always positive definite, the determinant of $\mathcal{C}_{1}$ will be $\left(\operatorname{Det}\left(D_{\theta}\right)\right)^{2}$ because element $(1,1)$ in $\mathcal{C}_{1}$ is 0 (a null matrix having the same dimensions as $D_{\theta}$.

From equation 3.79 , the system is observable, since the first two columns of $\mathcal{O}$ are identity. 


\subsection{Redundant robots}

In the case of kinematically redundant robots, cren if the global system if completely controllable and observable, the additional degrees of freedom may cause difficultics because the problem is underconstrained. It is then necessary to constrain the additional coordinates arising from the redundant degrees of freedom. for cxample by specifying additional impedance terms (see section A.2.1). Redundancy can also be used to minimize some criteria. The additional constraints to be added can be something simple, (c.g. with the Sarcos arm, controlling the position of the elbow), or something more sophisticated. In [26], the authors present a method to extend the task space using Lagrange multipliers.

\subsection{Error Characterization}

To be stable, computed torque techniques require the knowledge of the manipulator dynamics. Since a control law that does not completely inverse the roboi's dynamics will cause some error, it is important to characterize the nature of those errors and to describe them. We will first look at the steady state errors and then the transient crrors will be studied.

\subsubsection{Error on the stiffness term}

\section{Error from the control law}

When the transfer function was derived, we assumed small displacements and a lincarization was performed to map the Cartesian coordinates position into joint coordinates angles. As the robot moves away from its desired position the linearization no longer holds. The resulting error may vary depending on the robot configuration. 


$$
\begin{aligned}
\delta x & \approx J \delta \theta \\
\tau & =J^{T} F \\
F & =K_{r} \Delta x \\
\tau & \equiv J^{T} K_{x} \Delta x \\
\tau & \approx J^{T} K_{x} J \Delta \theta \\
\Delta x & =x-x_{d} \\
\Delta \theta & =\theta-\theta_{d} \\
\varepsilon & =\frac{J \Delta \theta-\left(\Lambda(\theta)-\Lambda\left(\theta_{d}\right)\right)}{\left(\Lambda(\theta)-\Lambda\left(\theta_{d}\right)\right)}
\end{aligned}
$$

From the previous equations, when $\left(\theta_{d}-\theta\right)$ is not small, the torque feedback (of the decoupler) coming from the joint angles error and multiplied by $J^{T} K_{x} . J$ contains an error and causes a steady state error on the stiffness term. The decoupler is no longer exact.

$$
K_{\text {effective }}=K_{\text {desired }} \frac{J \Delta \theta}{\left(\Lambda(\theta)-\Lambda\left(\theta_{d}\right)\right)}
$$

when $\left(\Lambda(\theta)-\Lambda\left(\theta_{d}\right)\right)$, is different from zero.

If a computed torque based method had been applied: $\tau=J^{T} \kappa_{x}\left(\Lambda(0)-\Lambda\left(0_{t}\right)\right)$ would be sent to the actuator and the problem would not occur. It is however possible to derive the correct expression for $K_{\theta}$, subtracting a corrective term:

$$
\begin{aligned}
K_{\theta} \Delta \theta & =J^{T} K_{x}\left(\Lambda(\theta)-\Lambda\left(\theta_{d}\right)\right) \\
K_{\theta} & =J^{T} K_{x}\left(\Lambda(\theta)-\Lambda\left(\theta_{d}\right)\right)\left(\left((\Delta \theta)^{T} \Delta \theta\right)^{-1}(\Delta \theta)^{T}\right)
\end{aligned}
$$

An alternative solution proposed in [33] allows us to nevertheless use analog PD controllers and eliminates any steady state error even for large $\Delta x$ in any direction. 
Since the cross coupling torgues are computed at each sample period, a geometrical projection of $X_{d}$ is used to calculate a correction on the desired joint angles.

When $\Delta x$ is large, the error arises from the approximation

$$
\Delta x \approx J \Delta 0
$$

To prevent the error, we simply incrementally update the desired joint space position $\theta_{d}$ at each servo cycle:

$$
\begin{aligned}
\tau & =J^{T} K_{x} \Delta x \\
\tau & =J^{T} K_{x} J\left(\theta_{\text {dnew }}-\theta\right) \\
\theta_{\text {dnew }} & =\theta+J^{-1} \Delta x
\end{aligned}
$$

Since equation 3.95 is computed at cach servo cycle, $\Delta x$ is small and this expression can also take care of the rotations. Given two positions in space, we have:

$$
\begin{aligned}
& T_{0}^{1}=\left(\begin{array}{cccc} 
& & & x_{d} \\
& R_{0}^{1} & & y_{d} \\
& & & z_{d} \\
0 & 0 & 0 & 1
\end{array}\right) \\
& T_{0}^{2}=\left(\begin{array}{llll} 
& & & x \\
& R_{0}^{2} & & y \\
& & & z \\
0 & 0 & 0 & 1
\end{array}\right) \\
& R=\left(R_{0}^{1}\right)^{-1} R_{0}^{2} \approx\left(\begin{array}{ccc}
1 & -r_{z} & r_{y} \\
r_{z} & 1 & -r_{x} \\
-r_{y} & r_{x} & 1
\end{array}\right) \\
& \Delta_{x}=\left(x_{d}-x, y_{d}-y, z_{d}-z, r_{x}, r_{y}, r_{z}\right)^{T}
\end{aligned}
$$




\section{Error caused by gravity}

The gravity has more influence on direct drive robots than on geared ones. It.s eflect is not reduced by the gear ratio. When the desired stiffuess is small or is of the same order of magnitude than the gravity components, the precision of the controller is affected, $\frac{x}{F_{d}} \neq K_{\text {desired }}$. Evidently when the desired stiffness is large, this crror reduces proportionally.

$$
\begin{aligned}
B_{\theta}\left(\dot{\theta}_{d}-\dot{\theta}\right)+K_{\theta}\left(\theta_{d}-\theta\right)+\hat{V}(0 . \dot{\theta})+\hat{G}(\theta)= & M(\theta) \vec{\theta}+V(\theta, \dot{\theta})+C(\theta) \\
M(\theta) \ddot{c}+B_{\theta} \dot{c}+K_{\theta} c= & M(0) \ddot{\theta}_{d}+V(0 . \dot{\theta})-\dot{V}(0 . \dot{\theta})+ \\
& G(\theta)-\dot{G}(\theta) \\
c_{\theta}= & K_{\theta}^{-1}(G(\theta)-\dot{C}(\theta))
\end{aligned}
$$

or in Cartesian coordinates,

$$
c_{x}=K_{x}^{-1}(G(x)-\dot{G}(x))
$$

\subsubsection{Error on the Damping term}

It was mentioned in section 3.2 that the off-diagonal terms of the inertia matrix produce couplings in the trarsient response and cause an error in the desired damping parameter. In this section, we will characterize and cstimate this error, and suggest the use of redundancy to minimize it. Although the proposed decentralized impedance controller does not require a dynamic model of the robot manipulator to remain stable, an estimation of the inertia matrix must be known to set the parameters of the transient response by fixing a desired damping ratio. Rewriting the transfer function equation from section 3.2 yields:

$$
\tau=\left(D_{\theta} s^{2}+B_{\theta} s+K_{\theta}\right) \theta
$$




$$
\begin{aligned}
\tau & =J^{T} F \\
F & =J^{-T}\left(D_{\theta} s^{2}+B_{\theta} s+K_{\theta}\right) J^{-1} x \\
& =\left(J^{-T} D_{\theta} J^{-1} s^{2}+B_{x} s+K_{r}\right) x \\
\text { Transfer functions } & =\left(D_{r} s^{2}+B_{x} s+K_{x}\right)^{-1}
\end{aligned}
$$

where

$$
D_{x}=J^{-T} D_{\theta} J^{-1}=\left(\begin{array}{ccc}
m_{x} & c_{1} & c_{2} \\
c_{1} & m_{y} & c_{3} \\
c_{2} & c_{3} & m_{z}
\end{array}\right)
$$

thus

$$
=\frac{\left(\begin{array}{ccc}
Y Z-c_{3}^{2} s^{4} & c_{2} \epsilon_{3} s^{4}-c_{1} Z s^{2} & c_{1} \epsilon_{3} s^{4}-c_{2} Y s^{2} \\
c_{2} \epsilon_{3} s^{4}-c_{1} Z s^{2} & X Z-c_{2}^{2} s^{4} & c_{1} \epsilon_{2} s^{4}-c_{3} X s^{2} \\
\epsilon_{1} c_{3} s^{4}-c_{2} Y s^{2} & c_{1} \epsilon_{2} s^{4}-\epsilon_{3} X s^{2} & X Y-c_{1}^{2} s^{4}
\end{array}\right)}{X Y Z-\left(\epsilon_{3}^{2} X+\epsilon_{2}^{2} Y+\epsilon_{1}^{2} Z\right) s^{4}+2 \epsilon_{1} \epsilon_{2} c_{3} s^{6}}
$$

where

$\mathrm{X}, \mathrm{Y}$ and $\mathrm{Z}$ are the Cartesian impedances in the $x, y$ and $z$ directions respectively.

The mathematical expression for the inertia matrix may contain many terms but its form is always that of equation 3.109. The off diagonal terms of equation 3.110 strictly depend on the $\epsilon_{i}$ 's of the inertia matrix. The couplings caused by the offdiagonal terms only affect the transient response and the error vanishes at steady state. Depending on the robot configuration, the $\epsilon_{i}$ 's of the inertia matrix will change and move the poles and zeros of the transfer function causing an error in the transient response. Stability is always preserved however (section 3.3), it is only a question of performance.

Looking at one Cartesian direction (e.g. $x$ ), it is possible to find an equivalent block diagram representating the transfer function.

$$
\frac{x}{f_{x}}=\frac{Y Z-\epsilon_{3}^{2} s^{4}}{X Y Z-\left(\epsilon_{3}^{2} X+\epsilon_{2}^{2} Y+\epsilon_{1}^{2} Z\right) s^{4}+2 \epsilon_{1} \epsilon_{2} \epsilon_{3} s^{6}}
$$




$$
=\frac{a+b}{a X+c}
$$

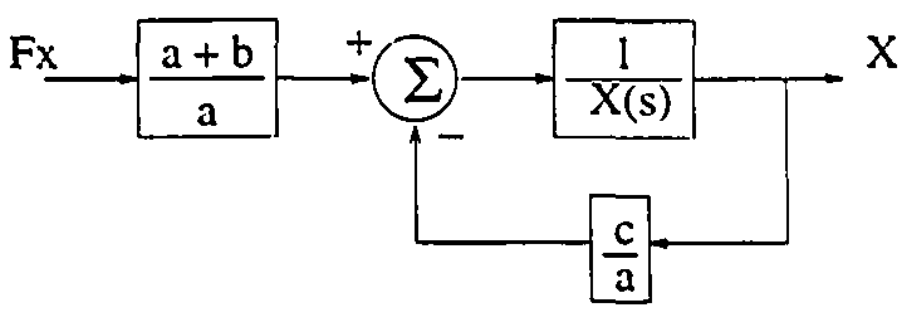

Figure 3.2: $A$ block diagram representation

We represent the coupling in the desired impedance as a prefilter and a feedback loop. From figure 3.2, the problem becomes obvious. As $b$ and $c$ decrease, the change in the poles and zeros due to the prefilter and feedback loop decreases and the transient response becomes closer to the ideal one.

By careful pose selection, it is possible to significantly reduce the of cliagonal terms of the inertia matrix. In order to estimate the error, we assume that the $m_{i}$ 's are large compared to the $c_{i}$ s such that equation $(3.110)$ can be rewritten in the following form,

$$
\begin{aligned}
\text { Tranfer functions } & =\left(M_{x} s^{2}+B_{x} s+K_{x}\right)^{-1} \\
& =\frac{\left(\begin{array}{ccc}
Y Z & -c_{1} Z s^{2} & -c_{2} Y s^{2} \\
-c_{1} Z s^{2} & X Z & -c_{3} X s^{2} \\
-c_{2} Y s^{2} & -c_{3} X s^{2} & X Y
\end{array}\right)}{X Y Z}
\end{aligned}
$$

Equation 3.114 means that we assume the error in the transient response comes from the coupling terms only and the influence of the $c_{i}$ 's on the poles and zcros of the impedance is neglected in a given direction. The transient res ${ }_{i}$ onse is now the superposition of the desired impedance transients and the coupling terms transients. 
In the case of a desired over damped impedance (usually the case in practical tasks) in each Cartesian coordinates direction, the time response of the denominator is given by,

$$
\begin{aligned}
\frac{1}{X Y Z} & =\frac{1}{\left(s+a_{x}\right)\left(s+b_{x}\right)\left(s+a_{y}\right)\left(s+b_{y}\right)\left(s+a_{z}\right)\left(s+b_{z}\right)} \\
f(t) & =C_{1} e^{-a_{x} t}+C_{2} c^{-b_{x} t}+C_{3} e^{-a_{y} t}+C_{4} c^{-b_{y} t}+C_{5} e^{-a_{z} t}+C_{6} e^{-b_{z} t}
\end{aligned}
$$

It is also possible to express the effect of the $c_{i}$ on the time domain transient response. The Laplace domain s operator is equivalent to a differentiation with respect to time, in the time domain. The impulse response is:

$$
\begin{aligned}
x(t) & =\left(C_{1} e^{-a_{x} t}+C_{2} e^{-b_{x} t}\right) f_{x}+\delta_{1}(t) f_{y}+\delta_{2}(t) f_{z} \\
y(t) & =\left(C_{3} c^{-a_{y} t}+C_{4} c^{-b_{y} t}\right) f_{y}+\delta_{1}(t) f_{x}+\delta_{3}(t) f_{z} \\
z(t) & =\left(C_{5} e^{-a_{z} t}+C_{6} e^{-b_{z} t}\right) f_{z}+\delta_{2}(t) f_{x}+\delta_{3}(t) f_{y} \\
\delta_{1}(t) & =-\epsilon_{1} \frac{d^{2}}{d t^{2}}\left(C_{1} e^{-a_{x} t}+C_{2} e^{-b_{x} t}+C_{3} e^{-a_{y} t}+C_{4} e^{-b_{y} t}\right) \\
\delta_{2}(t) & =-\epsilon_{2} \frac{d^{2}}{d t^{2}}\left(C_{1} e^{-a_{x} t}+C_{2} e^{-b_{x} t}+C_{5} e^{-a_{z} t}+C_{6} e^{-b_{z} t}\right) \\
\delta_{3}(t) & =-\epsilon_{3} \frac{d^{2}}{d t^{2}}\left(C_{3} e^{-a_{y} t}+C_{4} e^{-b_{y} t}+C_{5} e^{-a_{x} t}+C_{6} e^{-b_{z} t}\right)
\end{aligned}
$$

where

$$
\begin{aligned}
f_{i} & =\text { Impulse amplitude in the i direction } \\
a_{i}, b_{i} & =\text { Eigenvalues of the impedance in each direction } \\
C_{1} & =-\frac{1}{\left(a_{x}-a_{y}\right)\left(a_{x}-a_{z}\right)\left(a_{x}-b_{x}\right)\left(a_{x}-b_{y}\right)\left(a_{x}-b_{z}\right)} \\
C_{2} & =\frac{1}{\left(a_{x}-b_{x}\right)\left(-a_{y}+b_{x}\right)\left(-a_{z}+b_{x}\right)\left(b_{x}-b_{y}\right)\left(b_{x}-b_{z}\right)} \\
C_{3} & =\frac{1}{\left(a_{x}-a_{y}\right)\left(a_{y}-a_{z}\right)\left(a_{y}-b_{x}\right)\left(a_{y}-b_{y}\right)\left(a_{y}-b_{z}\right)}
\end{aligned}
$$




$$
\begin{aligned}
& C_{4}=\frac{1}{\left(a_{x}-b_{y}\right)\left(-a_{y}+b_{y}\right)\left(-a_{z}+b_{y}\right)\left(-b_{x}+b_{y}\right)\left(b_{y}-b_{z}\right)} \\
& C_{5}=\frac{1}{\left(a_{x}-a_{z}\right)\left(-a_{y}+a_{z}\right)\left(a_{z}-b_{x}\right)\left(a_{z}-b_{y}\right)\left(a_{z}-b_{z}\right)} \\
& C_{6}=\frac{1}{\left(a_{x}-b_{z}\right)\left(-a_{y}+b_{z}\right)\left(-a_{z}+b_{z}\right)\left(-b_{x}+b_{z}\right)\left(-b_{y}+b_{z}\right)} \\
& c_{i}=\text { off-diagonal terms of the Carlesian incrtia matrix }
\end{aligned}
$$

Given a desired impedance and a given location in space, the terms of equations $3.120,3.121$ and 3.122 become known and an expression for the transicnt crror can be computed finding the maximum of cach equations.

\subsection{Use of the Redundancy of Robots}

With redundant robots, it is possible to minimize the off cliagonal terms by selecting an optimal configuration of the joint angles for a given Cartesian position.

A well coordinated mass matrix reduces the cross coupling in the transient response and reduces the error between the desired damping ratio and the actual damping ratio.

In practice, redundancy cannot completcly decouple the inertia matrix but it can greatly affect the conditioning of the mass matrix as it is now shown.

Figure 3.3 shows the change of the mass matrix during a self motion (for a given Cartesian position in space). The three solid lines represent the three cliagonal terms of the mass matrix, $m_{x}, m_{y}, m_{z}$, and the dotted line represents the off diagonal terms of the mass matrix. In figure 3.4 , the optimization criterion proposed below is plotted as a function of a self motion parameters. For a given Cartesian coordiantes position in space, the optimization criterion will select the robot's configuration where its inertia matrix is best coordinated (as close as possible to the diagonal case). 


$$
\eta=\frac{\left|c_{1}\right|+\left|c_{2}\right|+\left|c_{3}\right|}{\left|m_{x}\right|+\left|m_{y}\right|+\left|m_{z}\right|}
$$

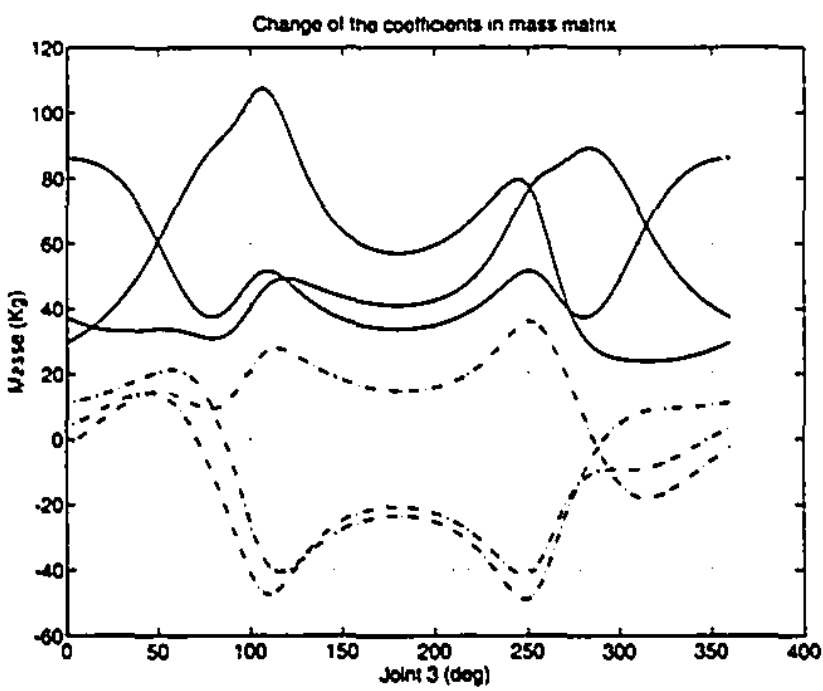

Figure 3.3: Mass matrix evolution vs redundant degree of freedom

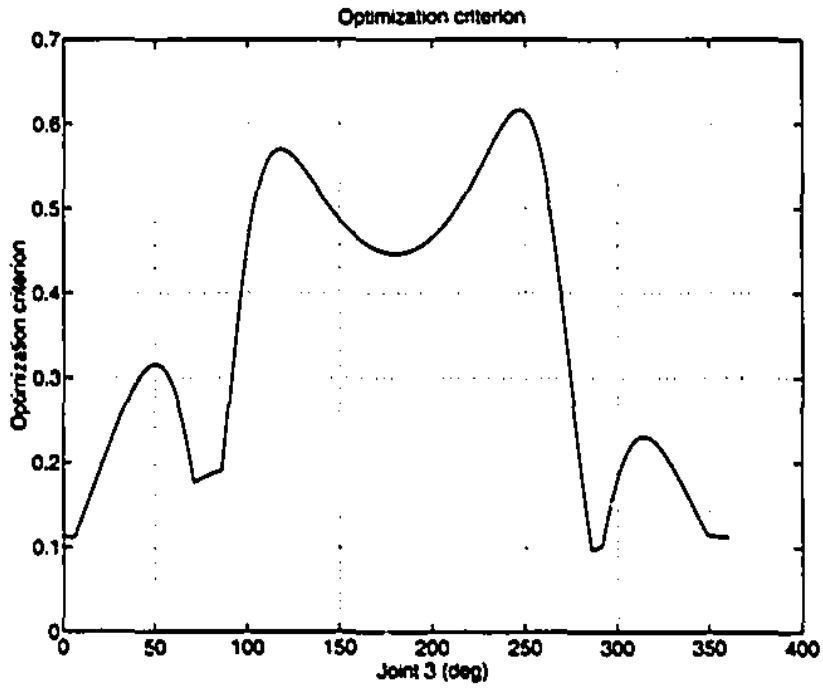

Figure 3.4: Optimization criteria vs redundant degree of freedom 


\subsection{Reachable Impedance}

The range of impedance that can be reached by the robot depends on mamy parameters. In this section, we will study different factors that may reduce the acheivable bandwidth of the resulting stable impedance.

\subsubsection{Unmodelled Flexibility}

Robot manipulators are often assumed to have rigid links, in practice it is never the case. The links have finite stiffness, although it can be made high. resulting in an increase in the order of the system [3]. Given the mass $(m)$ and stiffress $(k)$ of each link, an estimate of the resonant frequency is,

$$
\omega_{n_{\text {ref }}}=\sqrt{\frac{k}{m}}
$$

Equation 3.130 represents an upper bound for the range of reachable impedances. Given:

$$
Z_{x}(s)=M_{x} s^{2}+B_{x} s+K_{x}
$$

we have

$$
\begin{aligned}
& \omega_{n_{\text {denIred }}}=\sqrt{\frac{K_{x}}{M_{x}}} \\
& \omega_{n_{\text {deared }}} \leq \frac{\omega_{\text {res }}}{2}
\end{aligned}
$$

The bandwidth of the desired impedance cannot be higher than the natural frequency of the robot without causing problems in the control. 


\subsubsection{Sampling Pericd}

The sampling period of digital controllers will set an upper and a lower limit on the reachable impedances. This depends on the relations between the desired inertia. damping and stiffness $\left(M, B\right.$ and $\left.K^{\circ}\right)$. Intcrestingly, the sampling period places a lower limit on the reachable impedance. These conditions are expressed in equations 3.52 .3 .53 .3 .3 .54 .

\subsubsection{PD Controllers Maximum Gain}

The use of an analog (or hybrid) controller has several advantages, among them better robustness and stability. It is thus important to investigate their limits.

The analog controller implements the diagonal terms of the joint space impedance matrix. Those terms must be smaller than or equal to the maximum gains of the PD controller. In the following equations, the subscript max has been implied.

$$
\begin{aligned}
K_{\theta} & =J^{T} K_{x} J \\
K_{\theta_{11}} & =\sum_{n=1}^{N}\left(J_{n:}^{2}: K_{x_{n, n}}\right) \\
Q & =\left(\begin{array}{ccc}
J_{1,1}^{2} & \ldots & J_{1, n}^{2} \\
\vdots & & \vdots \\
J_{n, 1}^{2} & \ldots & J_{n, n}^{2}
\end{array}\right) \\
K_{\theta_{i 1}} & =Q K_{x}^{r} \\
K_{x} & =Q^{-1} K_{\theta_{1 i}}
\end{aligned}
$$

It is obvious from the previous equations that a given impedance in one direction will limit the impedance in another one. Thus a very stiff impedance may be obtained at the cost of a softer impedance in other directions. There is an explicit trade-off cxpressed by equation 3.138 . 


\subsubsection{Maximum Torque}

The impedance at a given point in space may be restricted by the three previous constraints but since it is a dynamic relation between a position and a force. then the actuator maximum saturation torque will limit the achievabie desired impedance. The limit is.

$$
\tau_{\max } \geq J^{T}\left(B_{x} s+K_{x}\right) \Delta x
$$

\subsubsection{Robot's Position}

In the case of non redundant robots, the reachable set of impedance at one point is unique. In the case of redundant robots. the reachable impedance at one point is not unique and redundancy may be used to change the limits on the reachable impedance.

\subsection{Simulation}

Before experiments were carried out on the Sarcos GRLA arm, simulations were performed to verify the performance of the proposed control algorithm under ideal conditions. The first part of this section will look at the steady state response and the second part will consider the transient response and how redundancy can improve the performance.

\subsubsection{Steady State Response}

A very slow force ramp is appiied at the end-effector of the manipulator causing

a deflection from the nominal position according to the desired stifiness, $\Delta x=\frac{F}{K}$. Figures 3.5 and 3.6 highlight the errors resulting from undecoupled decentralized control. In the presence of a small displacement, we see that the actual stiffress 
(slope of dotted line) does not match the desired stiffness (slope of solid line) when the deflection is large (figure 3.6). The effect of the various couplings is obvious. With a decoupler, figure 3.5 and 3.6, the response corresponds exactly to the ideal casc. An error arises from the linearization approximation $(x \approx J 0)$ causing a curve (dashed line) response even with decoupler. When the correction term is introduced to compensate for the torque feedback error, the response matches the ideal one (solid line). Equation 3.95 is solved in real time using Householder's method [29].
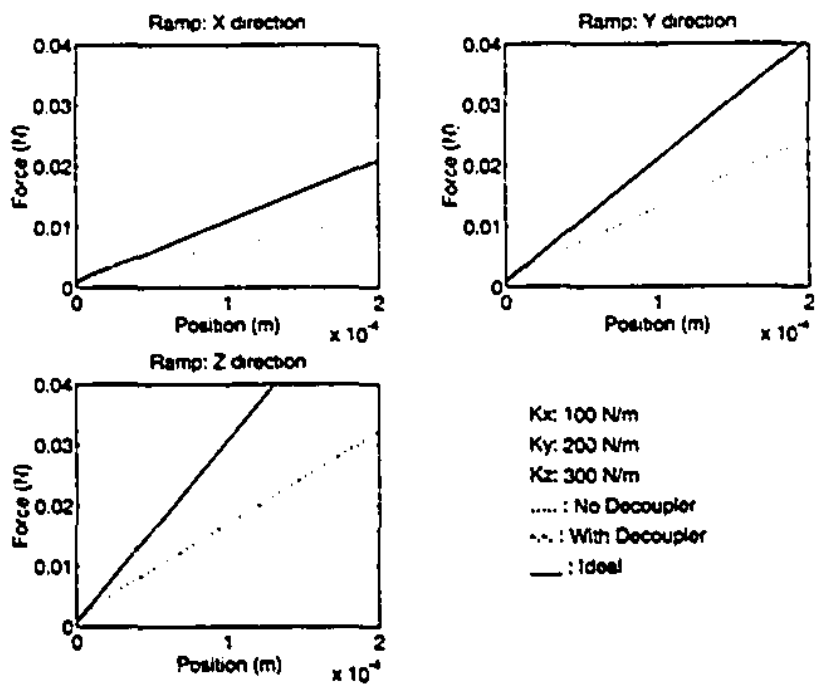

Figure 3.5: Small deflection

\subsubsection{Transient Response}

The main point here is to observe the effect of cross coupling on the transient response as well as the error on the damping factor. The simulations presented in figures $3.7,3.8$ and 3.9 consist, for each figure, of a step input of force applied at the end-effector of the robot along one of the Cartesian coordinates. The robot's transient response is then plotted. Ideally, there should be no response along the other direction. Depending on the degree of coupling of the inertia matrix, a transient may appear. 

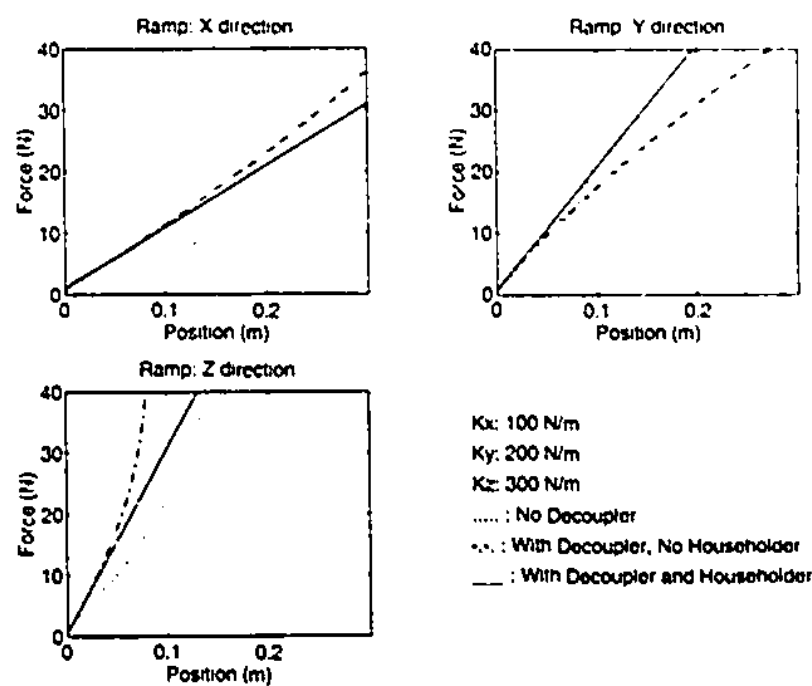

Figure 3.6: Large deflection

Since the arm under study is kinematically redundant, the effect of self motion on the transient response is studied. In figures $3.7,3.8$ and 3.9 , the dotted line is the ideal response, the dashed line corresponds to the worst situation and the solid line shows the best situation. We see that significant improvement is acheived when the inertia matrix is best conditioned. In some case it is even possible to completely remove the coupling transients. As seen on figures 3.8 and 3.9, the observed damping ratio corresponds almost perfectly to the desired one. In figure $3 . \bar{t}$, the damping ratio is slightly inacurate but the degree of decoupling is very good compared to a worst. case situation. 

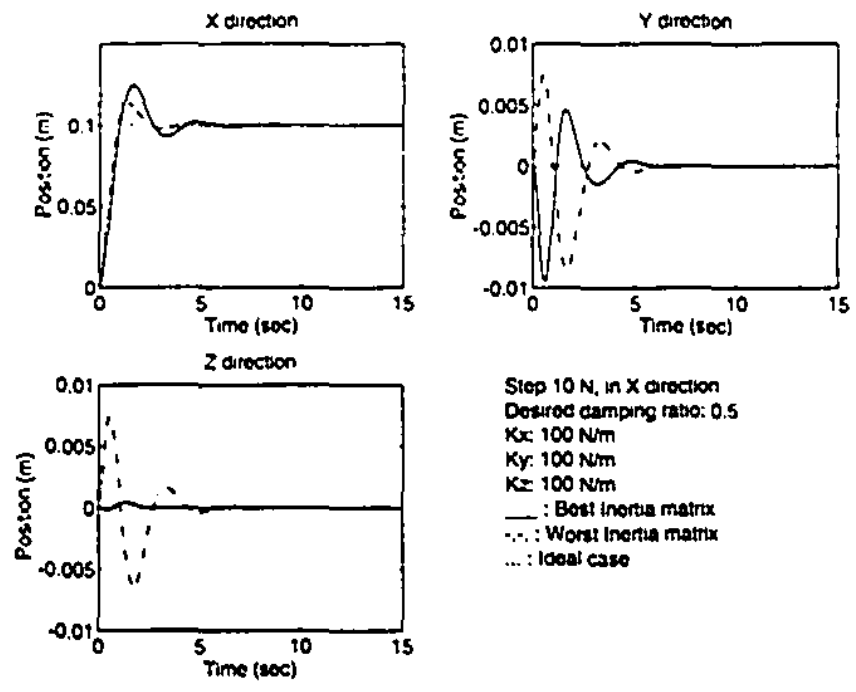

Slep $10 N, \ln \times$ arecton

Dosurec camping ratio: 0.5

$\mathrm{Kx}, 100 \mathrm{Nm}$

Kr. $100 \mathrm{Nm}$

$K y=100 \mathrm{Nm}$

_ : Bott inortis matrix

$\therefore$ : Wortl Inerta matrix

.... : looal caso

Figure 3. $\pi$ : Step in $\mathrm{X}$ direction
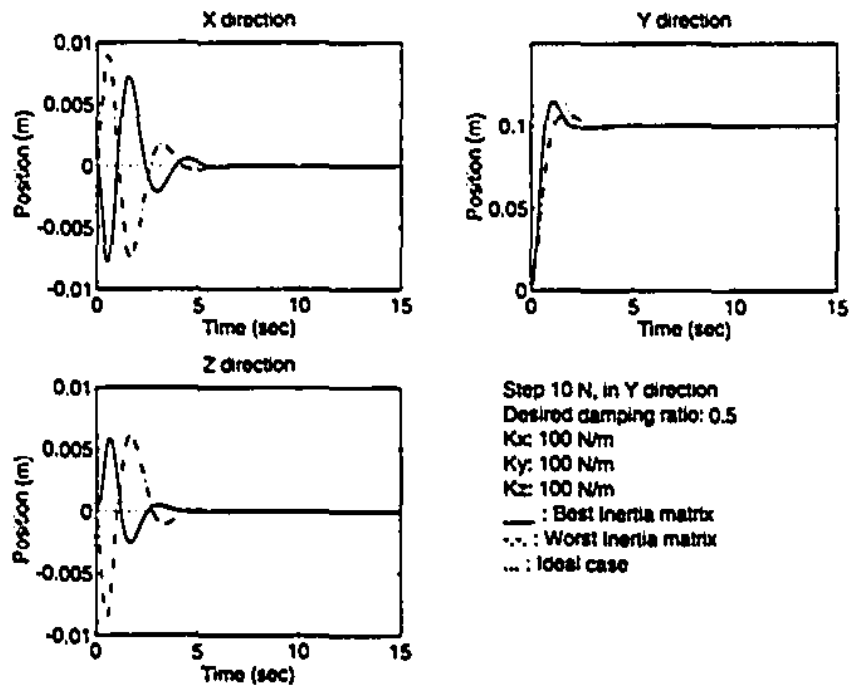

Figure 3.S: Step in $Y$ direction 

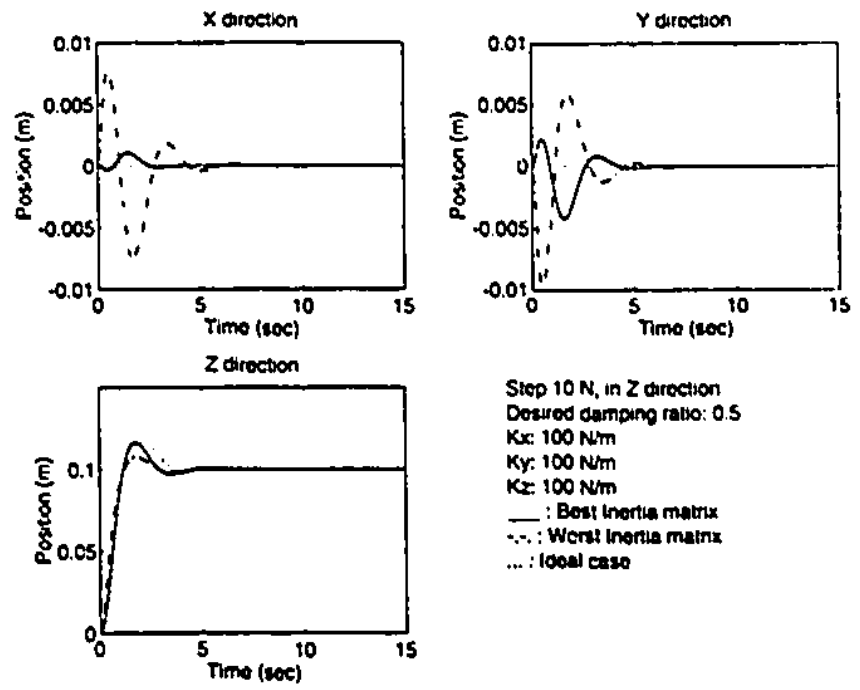

Step $10 \mathrm{~N}$, in Z direction Dosured demping ratio: 0.5

Kx: $100 \mathrm{Nm}$

Kr. $100 \mathrm{Nm}$

: Bost lnertia matrix

$\because-$ : Werti inorita mainx

... : looel caso

Figure 3.9: Step in Z direction 


\section{Chapter 4}

\section{Manipulator dynamics}

An expression for the inertia matrix in Cartesian coordinates is developed and is used to specify the cliaracteristics of the transient response. A gravity compensation algorithm is also developed. The ideal equation of motion of a manipulator resulting from the rigid body dynamics is given by,

$$
T=D_{\theta} \ddot{\theta}+V(\theta, \dot{\theta})+G(\theta)
$$

where $D_{\theta}, V(\theta, \dot{\theta})$ and $G(\theta)$ are respectively the mass matrix, centrifugal and coriolis components and the gravity component. In the static case, the gravity component $G(\theta)$ is the only contribution to the torque. In the dynamic case, both $D_{\theta}$ and $V(\theta, \dot{\theta})$ will affect the motion.

\subsection{Cartesian Inertia Matrix}

Four numbers are required to determine the mass properties of a link: the mass and three moments of inertia. The estimation of these parameters is difficult in practice because only the product of a mass by a length is observed and the extraction of the mass properties is not easy. 
In the proposed method, the robot manipulator is approximated by a distribution of point masses set at known locations on the arm. An estimate of the mass matrix is written as follows:

$$
\begin{aligned}
\tau & =\sum_{i=1}^{p}\left(J_{i}^{T} m_{i} g\right)=\Omega M \\
M & =\left(\Omega^{T} \Omega\right)^{-1} \Omega^{T} \tau=\left(\begin{array}{llll}
m_{1} & m_{2} & \ldots & m_{p}
\end{array}\right)
\end{aligned}
$$

The robot was moved to a number of locations and the joint angles and joint. torques were recorded producing a total of $3^{7}$ data points. Table 4.1 shows the mass estimates derived from this data for the Sarcos GRLA manipulator.

\begin{tabular}{|c|c|}
\hline$m_{i}$ & mass cstimate $(\mathrm{kg})$ \\
\hline$m_{1}$ & 7.3861 \\
\hline$m_{2}$ & 14.7723 \\
\hline$m_{3}$ & 24.6204 \\
\hline$m_{4}$ & 21.1916 \\
\hline$m_{5}$ & 17.7628 \\
\hline$m_{6}$ & 13.1910 \\
\hline
\end{tabular}

Table 4.1: Mass cstimate

Then the joint coordinates mass matrix is computed using the cstimates:

$$
D_{0}=\sum_{i=1}^{p}\left(J_{i}^{T} J_{i} m_{i}\right)
$$

The estimation of the Cartesian coordinates mass inatrix, $\mathcal{M}$, is derived from $D_{0}$. Assuming a small rate of change of the Jacobian with respect to time,

$$
\begin{aligned}
\dot{x} & =J \dot{\theta} \\
\ddot{x} & =J \ddot{\theta}+j \dot{\theta} \approx J \ddot{\theta}
\end{aligned}
$$




$$
\begin{aligned}
F & =\mathcal{U} \ddot{r} \\
\tau & =D_{\theta} \ddot{\theta} \\
\tau & =J^{T} F \\
\mathcal{U} & =J^{-T} D_{0} J^{-1}
\end{aligned}
$$

$D_{0}$ is a rather crude approximation since only six point masses have been taken into account. As will be demonst rated in chapter 5 , this estimate is surprisingly good.

\subsection{Gravity Compensation}

A model of the robot is used to calculate a gravity compensating torques. The complexity of the model may vary but at some point a model requiring the estimation of an excessively large number of parameter may not be necessarily better than another one using a few parameters (figure 4.1). Also, the robot's architecture may help reduce the number of parameters required for a good gravity compensation algorithm and at the same time, the computational cost to compute the compensating torque in real lime.

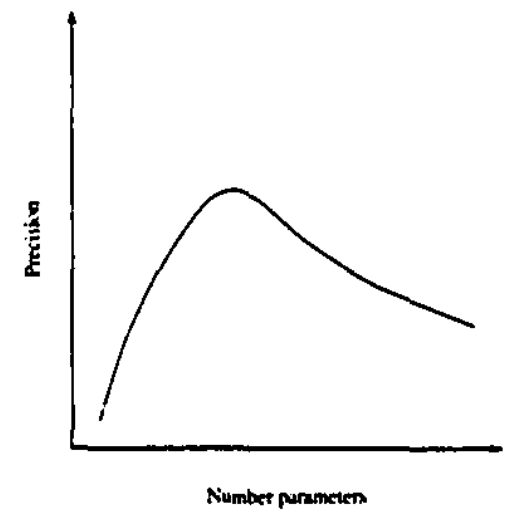

Figure 4.1: Precision vs number of parameters

A model requiring only six parameters has been developed for the Sarcos GRLA arm. We assumed that each center of mass was located along the manipulator link. 
The robot has been modelled with three point masses. $m_{1}$. $m_{2}$ and $m_{3}$ and three terms representing the point masses mult iplied by the ir center of masses, $m_{1} l_{1,}, m_{2} l_{12}$ and $m_{3} l_{r_{3}}$. 'The model developed in the previous section could have been used for gravity compensation but this one has a lower computational cost.

In figure 4.2 , the norm of the error between the estimate and actual gravity forec in Cartesian coordinates is presented. Considering that the Sarcos (BRI,A arm weights.s approximately $150 \mathrm{~kg}$, this simple model offers a good performance.

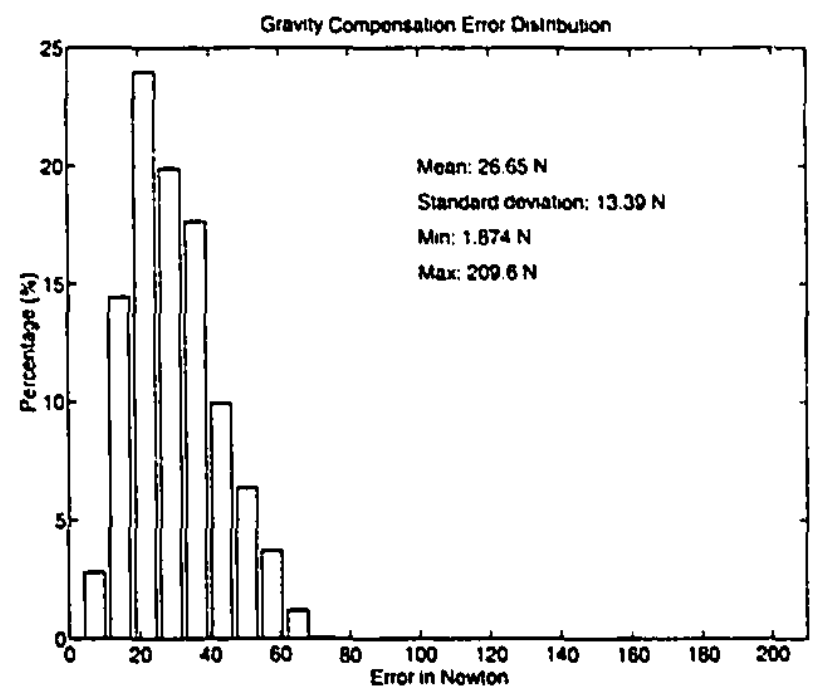

Figure 4.2: Gravity compensation 


\section{Chapter 5}

\section{Experimental Results}

\subsection{Experimental Setup}

The experimental setup consists of the Sarcos General Large Arm (GRLA) manipulator (figure 5.1). It uses hydraulic actuators driven with high performance jet pipe valves integrated at each actuators and strain gages are available at each joint to measure torque. This manipalator is characterized by a large workspace and a payload of approximately $100 \mathrm{~kg}$. Hydro-Québec uses it to develop telerobotics strategies to cveniually perform live line maintenance of electrical power lines. A Sparc CPU card inserted in a VME bus system is useci as a digital controller and is also used to communicate with the robot analog controller via a digital card from GreenSpring Computers. The robot is also supplied with a 3000 psi oil pressure.

\subsection{Implementation}

Given a desired position, the actual position of the manipulator is first read. Then, the Jacobian matrix is evaluated and the joint space gains (stiffness and rigidity) are derived. The diagonal terms of the joint space matrices are sent to the analog PD 


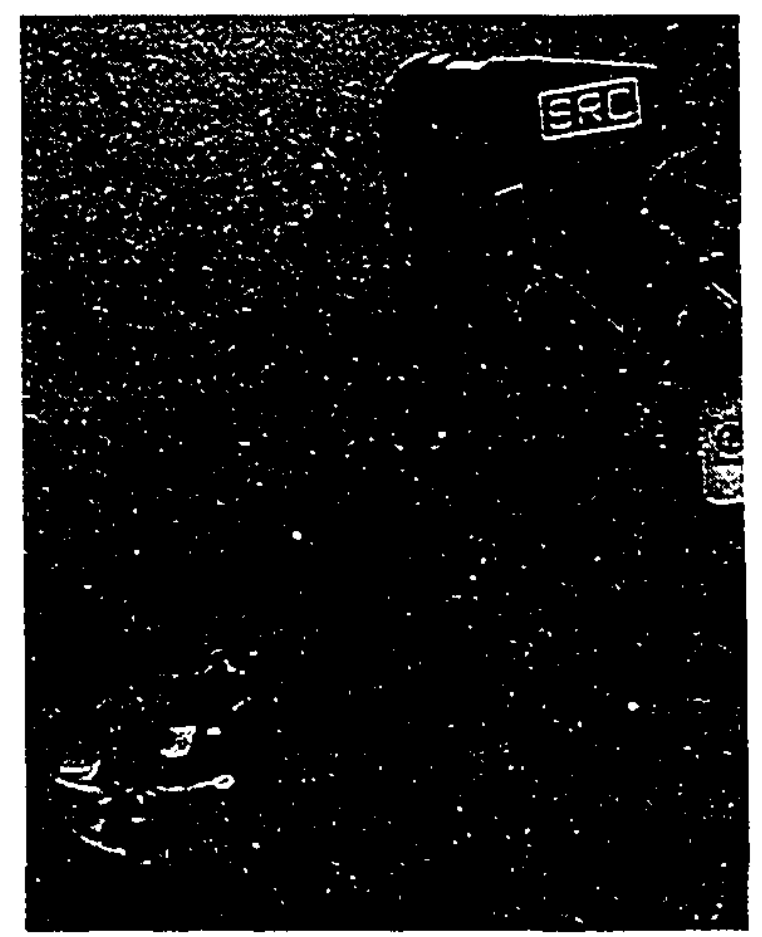

Figure 5.1: The Sarcos GRLA arm

controller and a cross-coupling torque is obtained from the off diagonal terms and the position error. To eliminate a steady state crror due to large deflections (section 3.T) a geometrical projection is used to produce a new desired position at cach cycle. Then the gravity compensation is computed. Finally, the cross-coupling, the gravity torques and the computed analog gains are written to the corresponding channels.

\subsection{Steady State Response}

A very slow ramp of force was applied at the end effector of the robot, causing a deflection. Ideally, the robot's displacement is given by,

$$
\Delta x=\frac{F}{K}
$$


where $F$ is the applied force and $K$ is the robot' stiffness.

Figures 5.2, 5.3. 5.4 show three different cases with the desired stiffnesses set to $1000 \mathrm{~N} / \mathrm{m}, 5000 \mathrm{~N} / \mathrm{m}, 10000 \mathrm{~N} / \mathrm{m}$ respectively. 'A known force is applied to the end effector of the manipulator and the joint displacements are recorded. The position of the end-effector is derived from this data.

The precision of the steady state response is limited by the precision of the gravity compensation algorithm. In the neighbourhood of the test configuration, the error of the gravity model has been compensated for as accurately as possible by adding an crror term derived from the discrepancy between the sensed torque and the gravity model torque. In this way, we take into account the $\Delta F$ due to the error on the gravity compensation algorithm.
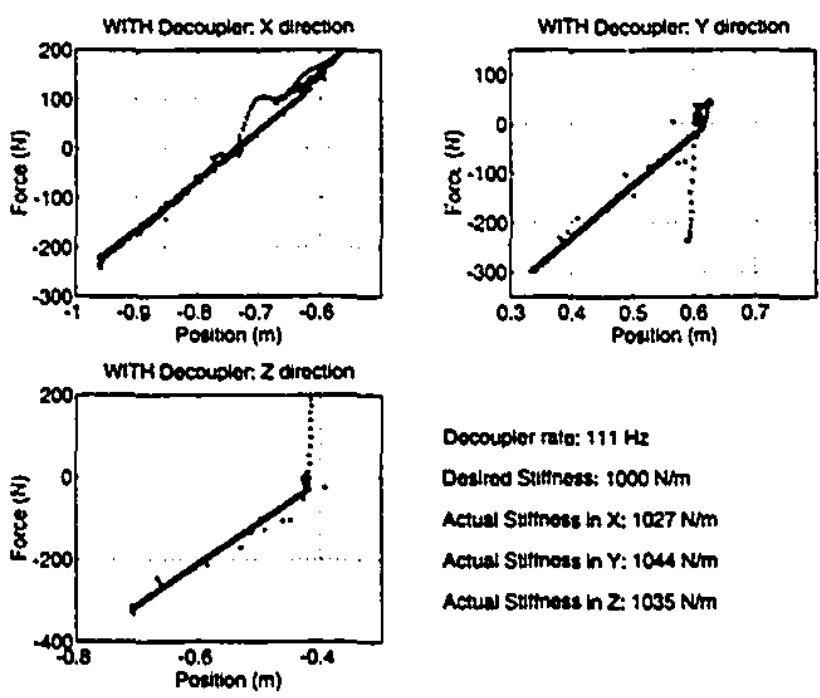

Decouplor fato: 111 Hs

Deslrou Suthess: $1000 \mathrm{~N} / \mathrm{m}$

Actual Stithose $\ln x: 1027 \mathrm{Nm}$

Actual Surtinoss in Y: $10 \mathrm{~m} \mathrm{Nm}$

Actur Sutmess in Z: $1035 \mathrm{Nm}$

Figure 5.2: Steady state with decoupler, $1000 \mathrm{~N} / \mathrm{m}$

The estimation of the stiffness from data plotted on figures $5.2,5.3,5.4$ was done using a least squares method. Table 5.3 shows the percentile of error between the desired and the estimated stiffness. The most important source of error is due to the

\footnotetext{
${ }^{1} 10000 \mathrm{~N} / \mathrm{m}$ corresponds to $1 \mathrm{~kg}$ per $\mathrm{mm}$
} 

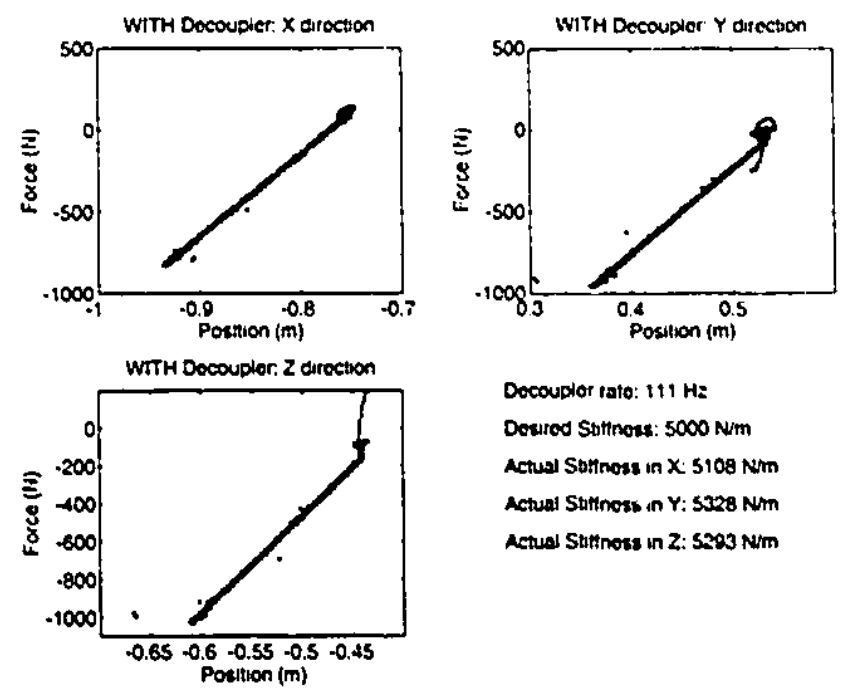

Docouplor rate: $111 \mathrm{HE}$ Deared Stitrous: $5000 \mathrm{Nm}$ Actud Satinoss in X: $5108 \mathrm{Nm}$ Actual Stittnoss in Y: $5328 \mathrm{~N} / \mathrm{m}$ Actual Suttnoss in 2: $5203 \mathrm{~N} / \mathrm{m}$

Figure 5.3: Steady state with decoupler, $5000 \mathrm{~N} / \mathrm{m}$

\begin{tabular}{|c|c|c|c|}
\hline Stiffness $(\mathrm{N} / \mathrm{m})$ & Error in $\times(\%)$ & Error in $\mathrm{y}(\%)$ & Error in $\%(\%)$ \\
\hline 1000 & 2.7 & 4.4 & 3.5 \\
\hline 5000 & 2.2 & 6.6 & 5.9 \\
\hline 10000 & 0.5 & 5.9 & 6.0 \\
\hline
\end{tabular}

Table 5.1: Desired stiffness error

presence of transients at the beginning of the data acquisition and the rest is due to noise in the measurement.

Figure 5.5 corresponds to the case of pure PD control where the PD gains are continuously updated in order to meet the impedance but no decoupler is applied. In section 3.1 , we saw that such a problem is under determined since the number of parameters to set is larger than the control variables. The results using the complete control algorithm are presented on figure 5.6. The performance degrades when the robot is moved away from its nominal position because of the linear approximation made with the Jacobian matrix (section 3.7). In theory, it was shown that the precision in fact, degrades. In practice, the system may escape control altogether. Finally, 

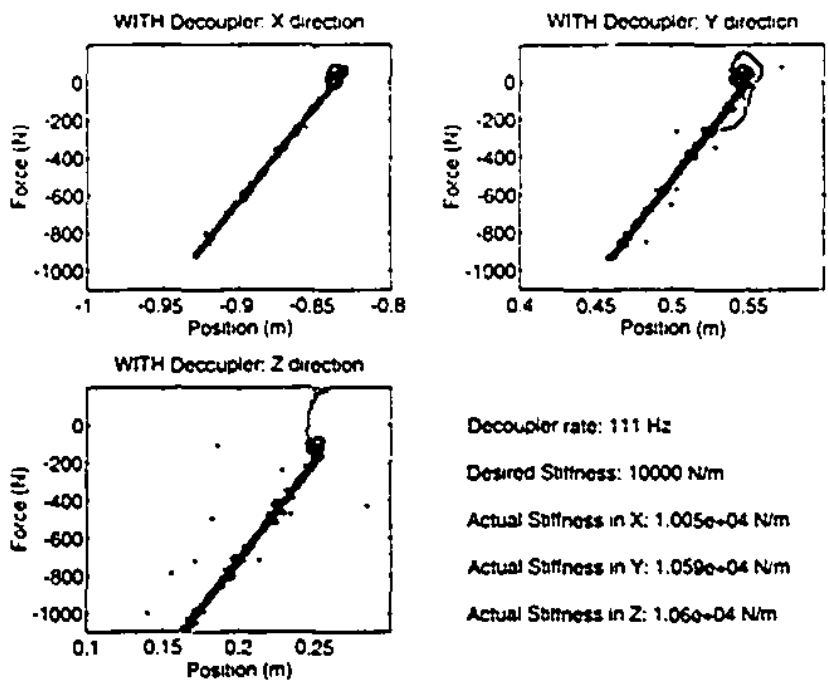

Decoudor rate: i: $\mathrm{Hz}$

Dosured Suttross: $10000 \mathrm{Nm}$

Actual Suifinoss in $\mathrm{X}: 1,0050+04 \mathrm{Nm}$

Actual Suftness in Y: $1.050000 \mathrm{Nim}$

Actual Solness in Z: 1.060004 N/m

Figure 5.4: Steady state with decoupler, $10000 \mathrm{~N} / \mathrm{m}$

the decoupler is added in conjunction with the geometrical projection to compensate for the lincarization approximation (sec figure 5.7). 

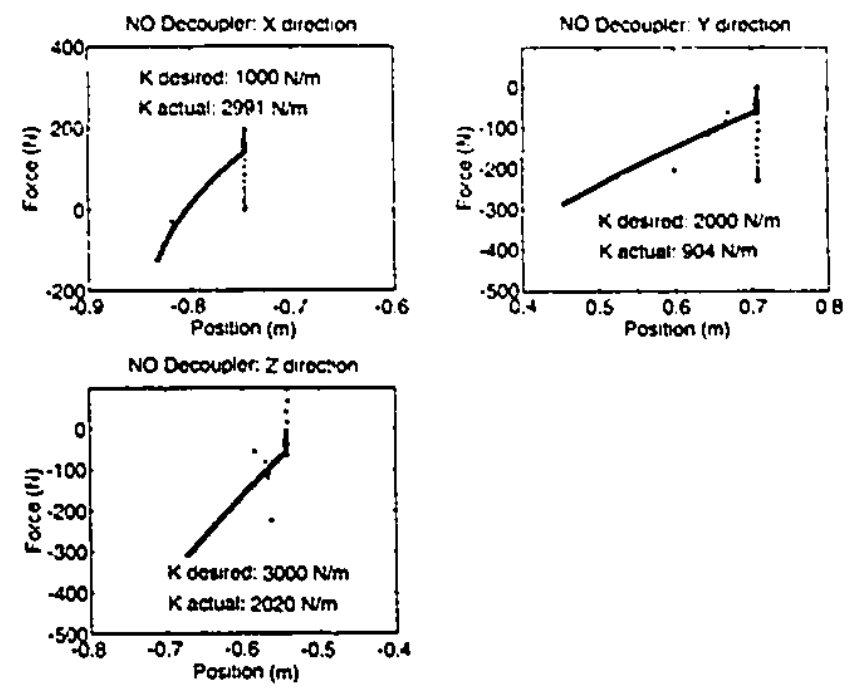

Figure 5.5: Steady state no decoupler, 1000, 2000, $3000 \mathrm{~N} / \mathrm{m}$
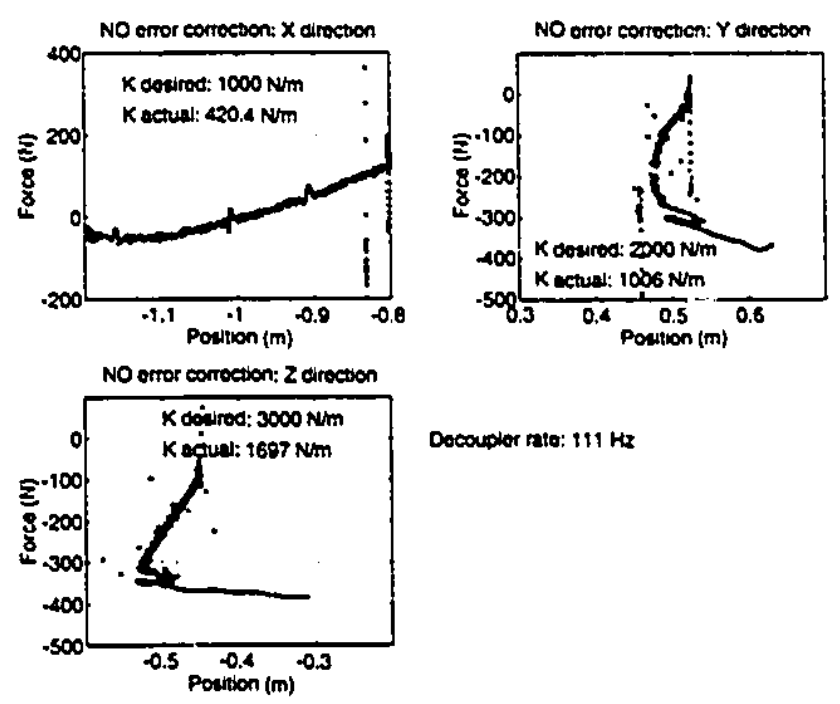

Decoupler rato: $111 \mathrm{~Hz}$

Figure 5.6: Steady state no error correction, $1000,2000,3000 \mathrm{~N} / \mathrm{m}$ 

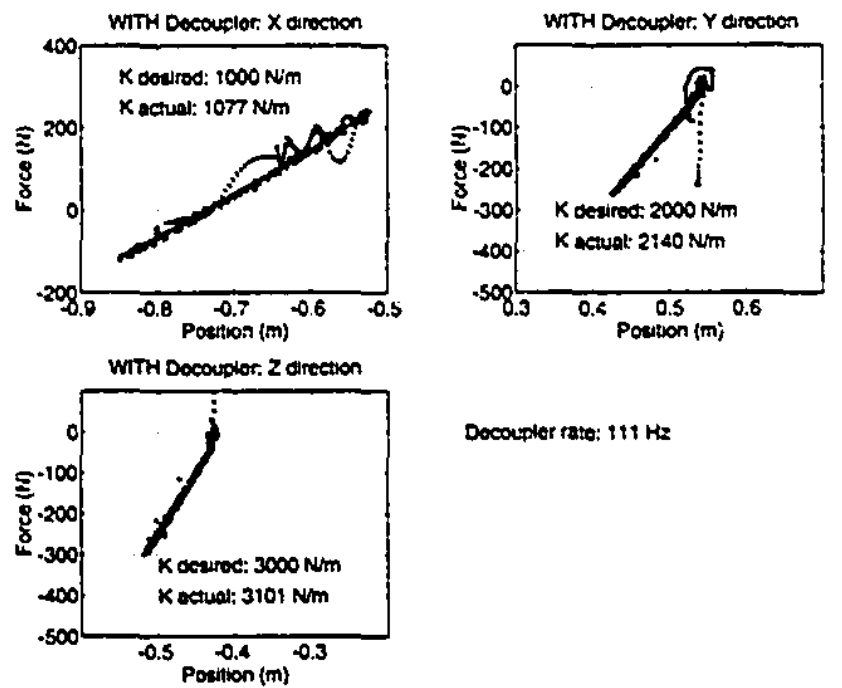

Docoupler rate: iti Hz

Figure 5.7: Steady state with decoupler, 1000, 2000, $3000 \mathrm{~N} / \mathrm{m}$ 


\subsection{Transient Response}

An cstimate of the manipulator inertia matrix is needed to program the transient response characteristics. In chapter 4, we developed a method to estimate the value of this matrix. For the purpose of this section. we assume that the transfer function in a given direction is,

$$
\begin{aligned}
\frac{X}{F} & =\frac{1}{m s^{2}+b s+k} \\
\zeta & =\frac{b}{2 \sqrt{m k}}
\end{aligned}
$$

We experimentally checked that the response along a given direction matches the desired one. We also checked for the absence of coupling accross the other directions. Inspection of the figures below shows that the results are quite convincing given the numerous sources of uncertainties in such a complex system. The steady state errors are caused by the error of gravity compensation model.
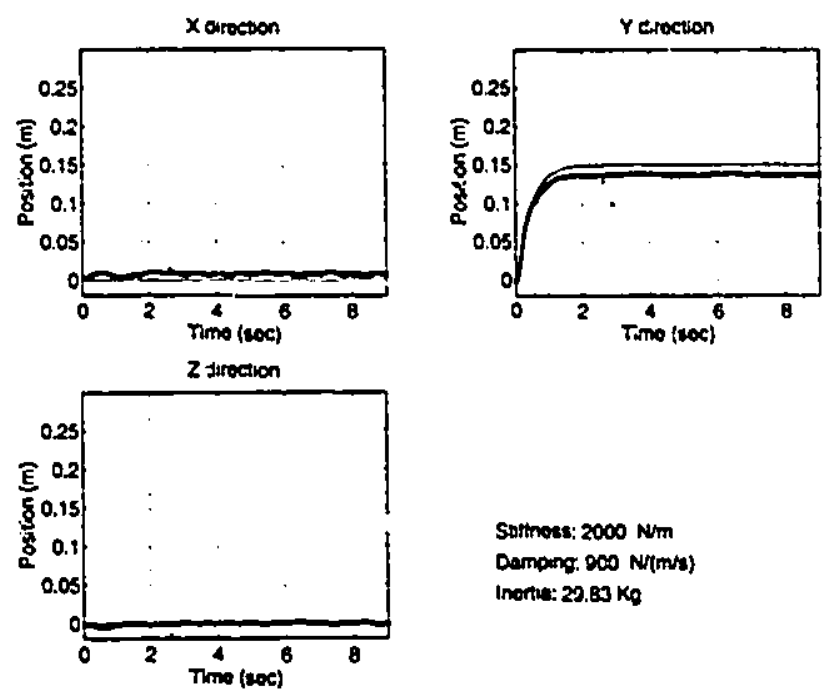

Figure 5.8: Transient Response 

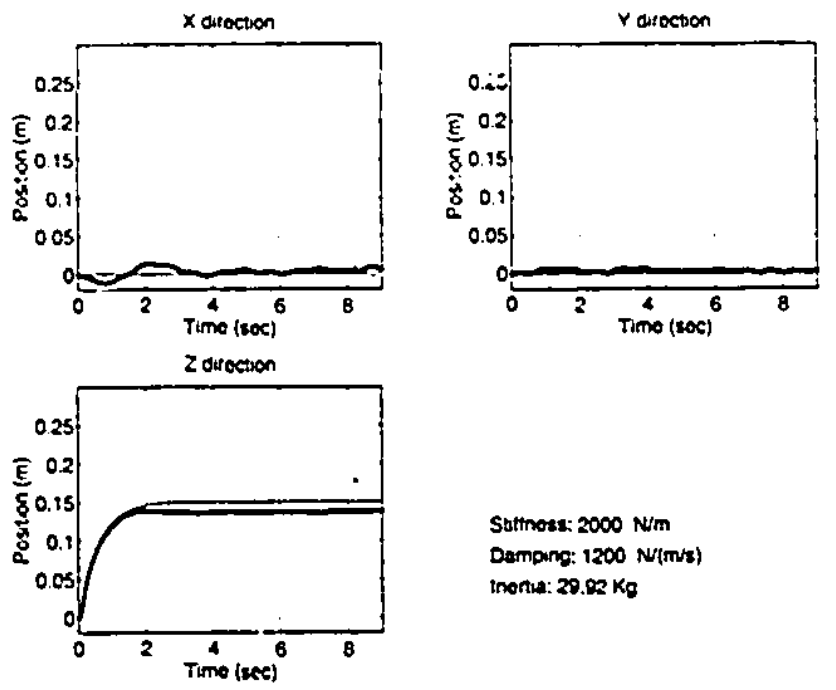

Suttnoes: $2000 \mathrm{~N} / \mathrm{m}$ Damping: $1200 \mathrm{~N}(\mathrm{~m} / \mathrm{s})$ thortua: $29.02 \mathrm{Kg}$

Figure 5.9: Transient Response
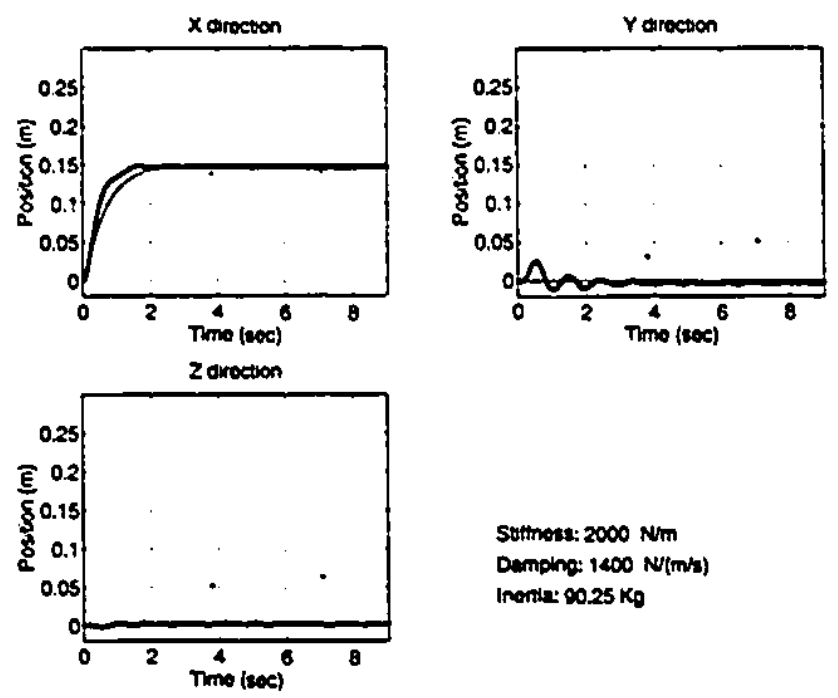

Suthots: $2000 \mathrm{Nm}$
Demplng: $1400 \mathrm{~N} / \mathrm{m} / \mathrm{s})$
Inemis: $00.25 \mathrm{Kg}$

Figure 5.10: Transient Response 

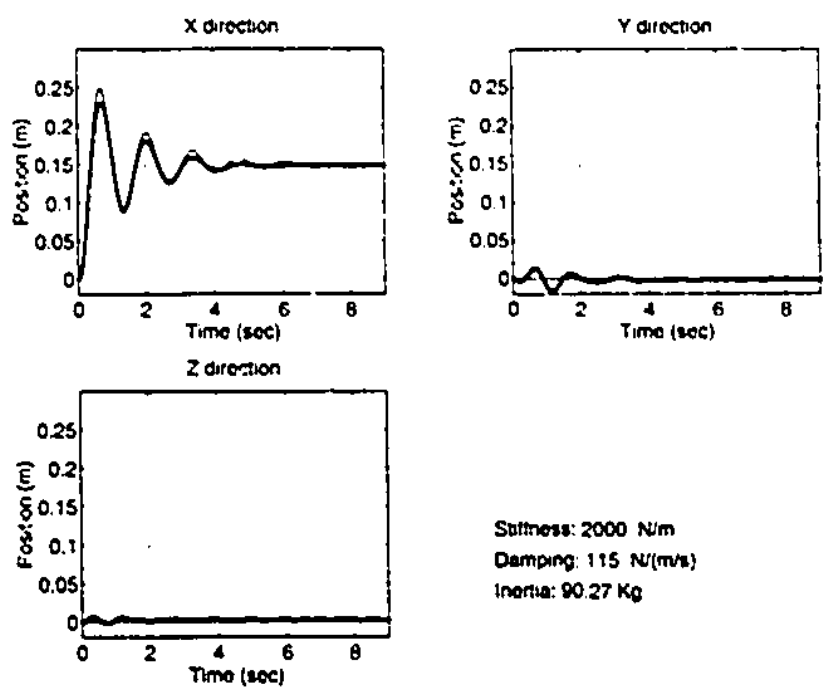

Sutthess: $2000 \mathrm{Nm}$

Demping: $115 \mathrm{~N}(\mathrm{~m} / \mathrm{n})$

Inortia: $90.27 \mathrm{Ko}$

Figure 5.11: Transicnt Response
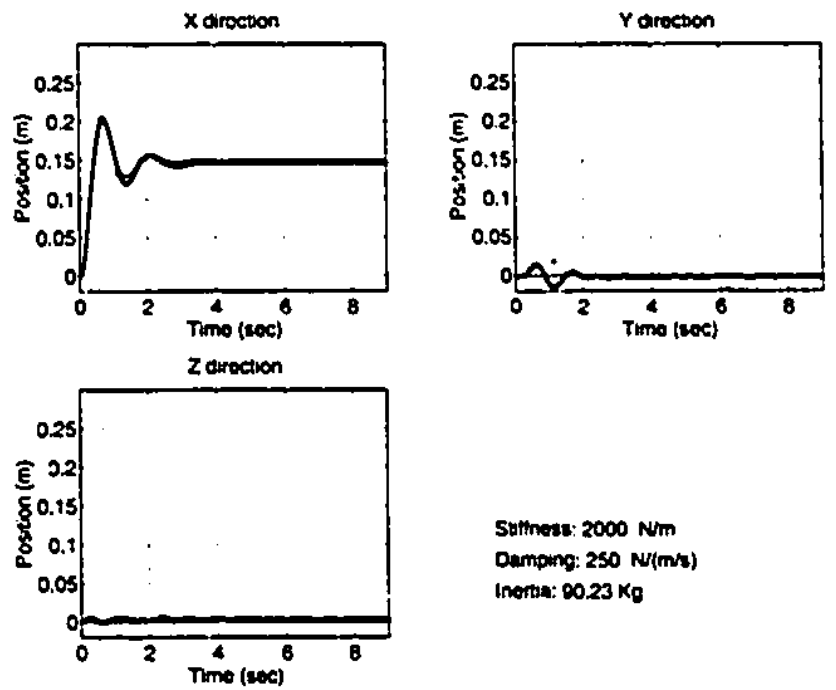

Sthous: $2000 \mathrm{~N} / \mathrm{m}$ Dumping: $250 \mathrm{~N}(\mathrm{~m} / \mathrm{s})$

Inerta: $00.23 \mathrm{Kg}$

Figure 5.12: Transient Response 

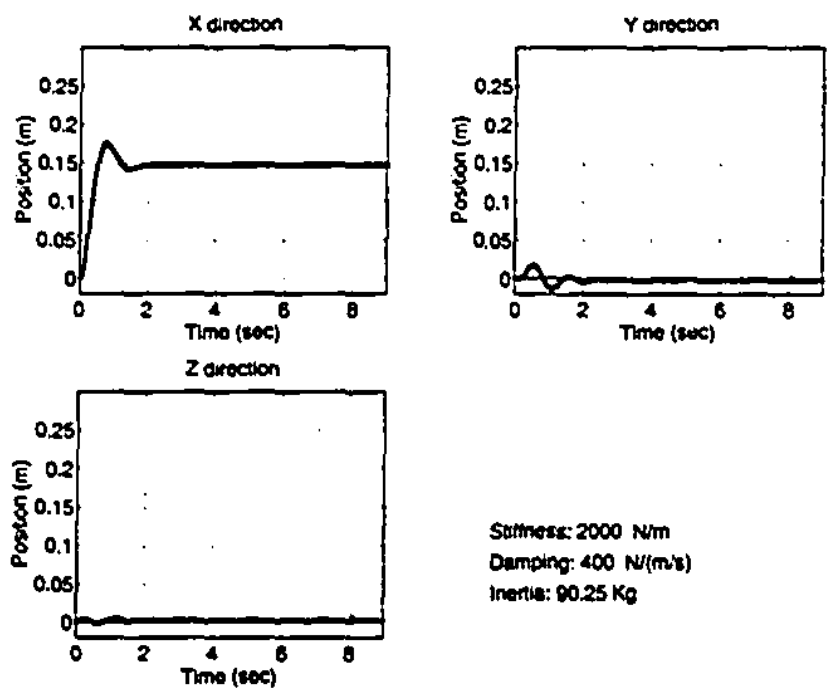 Damping: $400 \mathrm{~N} / \mathrm{mir}$ ) Inetis: $00.25 \mathrm{Ko}$

Swimers: $2000 \mathrm{~N} / \mathrm{m}$

Figure 5.13: Transient Response 


\subsection{Contact Tasks}

The implementation proposed in this thesis uses co-located torque sensors instead of a force sensor placed at the end-effector of a manipulator. The advantage of using (o)located torque sensors is obvious when contact tasks are performed. Since the sensors are placed at each actuators, the link dynamics as well as the sensor dymamics do not. deteriorate the force feedback information but is just seen as a perturbation, loading to a very stable response when the manipulator contacts a stiff surface. Figure 5.14 present the situation where the robot follows a desired trajectory and then hits a stiff wall placed along the path. The desired and followed trajectories have been plotted. While the manipulator is in contact with the wall, the position in the $Z$ direction remains the same and a force arises corresponding to the product of the desired stiffness by the distance between the desired and followed trajectory. The desired stiffress has bcen sele ted to be $4000 \mathrm{~N} / \mathrm{m}$ and th: damping was set to $250 \mathrm{~N} /(\mathrm{m} / \mathrm{s})$. The maximum applied force is $434 \mathrm{~N}$ when the difference between the desired and followed trajectory is $0.1004 \mathrm{~m}$. An error of $8.25 \%(33 \mathrm{~N})$ is due to the error on the gravity compensation model (see figure 4.2 ). 

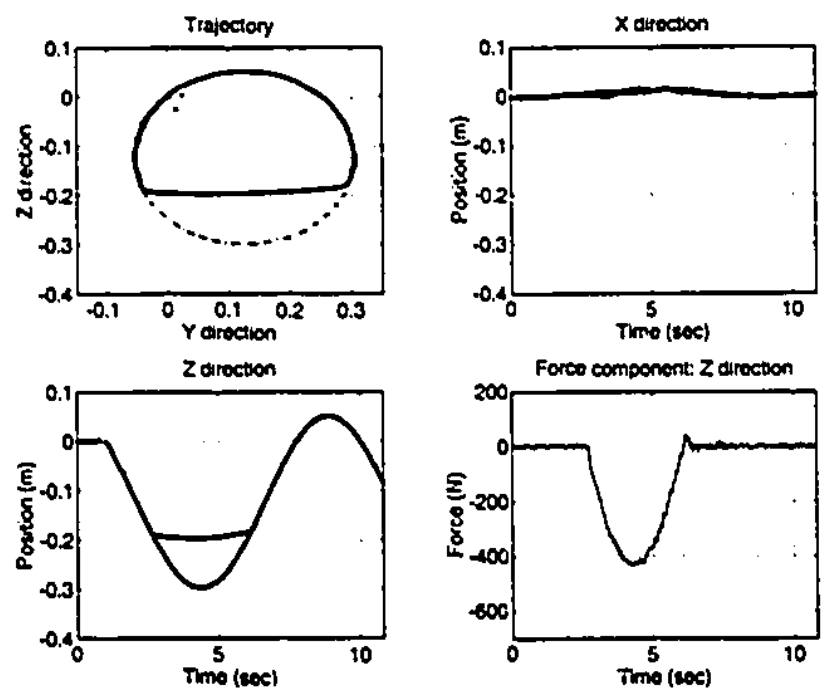

Figure 5.14: Contact task 


\section{Chapter 6}

\section{Conclusion}

The simpler the control is, the better its properties can be demonstrated: theoretically, practically and experimentally, in terms of stability, performance, robustuess and generality. In this thesis, a simple theory leading to the implementation a joint. space controller with decoupled impedance in Cartesian coordinates was presented. The proposed controller is based on joint variables $(0, \tau)$ and does not require complex changes of coordinates.

It has been found that steady state errors may appear depending on the precision of the gravity compensation algorithm. Also a correction has been proposed to overcome the steady state error on the impedance arising when the robot is far from its desired position. Since the Cartesian inertia matrix is not diagonal, coupling in the transient response is created. The error terms have been expressed and are directly proportional to the importance of the off diagonal terms in the inertia matrix. They are bounded and in practice quite small. When redundant robots are used, it is possible to position the robot such that the off diagonal terms are minimized and thus reduce the transient error.

Stability in continuous time has been proven as well as in the digital case where stability conditions have been expressed. The decentralized impedance controller 
has been implemented on a redundant robot, the Sarcos (GRLA) arm available at Institut de Recherche d'Hydro-Québec and experimental results have been presented for various situations. In addition, the decentralized impedance control technique that has been proposed, owing to intrinsinc robustness, is easy to tune. Sloppy tuning will degrade performance but stability will remain unconditional, provided that simply expressed bounds on the gains are not exceeded. This is an important property since the robotic equipment is to be used in the field where highly qualified personel is not necessarily available. 


\section{Appendix A}

\section{Kinematics}

This chapter discusses the kinematics of the Sarcos (GRLA) arm. 'This is required to implement the decentralized impedance controller. The forward kinematics, the Jacobian matrix and one method to compute the inverse kinematics of the redundant robot are presented.

The Sarcos arm that is used in the implementation, is a seven degree of freedom, zero offset anthropomorphic manipulator.

\section{A.1 Forward Kinematics}

\section{A.1.1 Denavit-Hartenberg Parameters}

Table A.1.1 shows the Denavit-Hartenberg parameters for this manipulator. It is followed by the transform matrices and the Jacobian matrix.

\section{A.1.2 Transformation matrices}

Using the Denavit-Hartenberg parameters, we can compute the transformations mitrices relating one frame to the next. 


\begin{tabular}{|c|c|c|c|c|}
\hline$i$ & $a_{i}$ & $\alpha_{i}$ & $d_{i}$ & $0_{i}$ \\
\hline 1 & 0 & $\frac{\pi}{2}$ & 0 & $\theta_{1}$ \\
\hline 2 & 0 & $\frac{\pi}{2}$ & 0 & $0_{2}$ \\
\hline 3 & 0 & $\frac{-\pi}{2}$ & $l_{1}$ & $\theta_{3}$ \\
\hline 4 & 0 & $\frac{\pi}{2}$ & 0 & $0_{4}$ \\
\hline 5 & 0 & $\frac{-\pi}{2}$ & $l_{2}$ & $0_{5}$ \\
\hline 6 & 0 & $\frac{\pi}{2}$ & 0 & $\theta_{6}$ \\
\hline 7 & $-l_{3}$ & $\pi$ & 0 & $0_{4}$ \\
\hline
\end{tabular}

Table A.1: Slave arm Denavit-Hartenberg parameters

$$
T_{i}^{i-1}=\operatorname{Rot}_{z}\left(\theta_{i}\right) \operatorname{Trans}_{z}\left(d_{i}\right) \operatorname{Trans}_{x}\left(a_{i}\right) \operatorname{Rot}_{x}\left(\alpha_{i}\right)
$$

thus

$$
T_{i}^{i-1}=\left(\begin{array}{cccc}
c_{\theta_{1}} & -s_{\theta_{i}} c_{a l p h a,} & s_{\theta_{1}} s_{\alpha l p h i a_{1}} & c_{t h e t a_{i}} a_{i} \\
s_{\theta_{i}} & c_{\theta_{i}} s_{\alpha_{1}} & -c_{\theta_{1}} s_{\alpha_{i}} & s_{\theta_{i}} a_{i} \\
0 & s_{\alpha_{1}} & c_{\alpha_{i}} & d_{i} \\
0 & 0 & 0 & 1
\end{array}\right)
$$

where

$a_{i}=$ distance along $x_{i}$ from $o_{i}$ to the intersection of the $x_{i}$ and $z_{i-1}$ axes.

$\alpha_{i}=$ the angle between $z_{i-1}$ and $z_{i}$ measured about $x_{i}$.

$d_{i}=$ the distance along $z_{i-1}$ form $o_{i-1}$ to the intersection of the $x_{i}$ and $z_{i-1}$ axes.

$\theta_{i}=$ the angle between $x_{i-1}$ and $x_{i}$ measured about $z_{i-1}$.

$$
T_{1}^{0}=\left(\begin{array}{cccc}
c_{1} & 0 & s_{1} & 0 \\
s_{1} & 0 & -c_{1} & 0 \\
0 & 1 & 0 & 0 \\
0 & 0 & 0 & 1
\end{array}\right)
$$




$$
\begin{aligned}
& T_{2}^{1}=\left(\begin{array}{cccc}
c_{2} & 0 & s_{2} & 0 \\
s_{2} & 0 & -c_{2} & 0 \\
0 & 1 & 0 & 0 \\
0 & 0 & 0 & 1
\end{array}\right) \\
& T_{3}^{2}=\left(\begin{array}{cccc}
c_{3} & 0 & -s_{3} & 0 \\
s_{3} & 0 & c_{3} & 0 \\
0 & -1 & 0 & l_{1} \\
0 & 0 & 0 & 1
\end{array}\right) \\
& T_{4}^{3}=\left(\begin{array}{cccc}
c_{1} & 0 & s_{4} & 0 \\
s_{1} & 0 & -c_{4} & 0 \\
0 & 1 & 0 & 0 \\
0 & 0 & 0 & 1
\end{array}\right) \\
& T_{5}^{4}=\left(\begin{array}{cccc}
c_{5} & 0 & -s_{5} & 0 \\
s_{5} & 0 & c_{5} & 0 \\
0 & -1 & 0 & l_{2} \\
0 & 0 & 0 & 1
\end{array}\right) \\
& T_{6}^{5}=\left(\begin{array}{cccc}
c_{6} & 0 & s_{6} & 0 \\
s_{6} & 0 & -c_{6} & 0 \\
0 & 1 & 0 & 0 \\
0 & 0 & 0 & 1
\end{array}\right) \\
& T_{i}^{6}=\left(\begin{array}{cccc}
c_{i} & s_{i} & 0 & 0 \\
s_{i} & -c_{i} & 0 & 0 \\
0 & 0 & -1 & 0 \\
0 & 0 & 0 & 1
\end{array}\right)
\end{aligned}
$$

By forming $T_{7}^{0}$ we easily obtain the Cartesian positions, $x, y$ and $z$. From the structure of the robot, only the first four links will produce a position displacement, so: 


$$
\begin{aligned}
& x=l_{1} c_{1} s_{2}+l_{2}\left(c_{1} c_{4} s_{2}+\left(c_{1} c_{2} c_{3}+s_{1} s_{3}\right) s_{4}\right) \\
& y=l_{1} s_{1} s_{2}+l_{2}\left(c_{4} s_{1} s_{2}+\left(c_{2} c_{3} s_{1}-c_{1} s_{3}\right) s_{4}\right) \\
& z=-l_{1} c_{2}+l_{2}\left(-c_{2} c_{4}+c_{3} s_{2} s_{4}\right)
\end{aligned}
$$

Where $c_{i}$ and $s_{i}$ represent respectively $\cos \left(q_{i}\right)$ and $\sin \left(q_{i}\right) . l_{1}$ is $0.8788 \mathrm{~m}$. and $l_{2}$ is $0.7620 \mathrm{~m}$. The joint limits for this arm are given by table A.1.2.

\begin{tabular}{|c|c|c|}
\hline$q_{i}$ & $q_{i_{\min }}$ & $q_{i_{\max }}$ \\
\hline$q_{1}$ & -10.0 & 80.0 \\
\hline$q_{2}$ & 10 & -100 \\
\hline$q_{3}$ & -90 & 90 \\
\hline$q_{i}$ & 30 & 140 \\
\hline$q_{5}$ & 0 & 150 \\
\hline$q_{6}$ & -90 & 90 \\
\hline$q_{i}$ & -45 & 45 \\
\hline
\end{tabular}

\begin{tabular}{|c|c|}
\hline Denavit-Hartenberg & $\overline{\operatorname{Sarcos}}$ \\
\hline$\theta_{1 D H}$ & $-\frac{\pi}{2}+\theta_{1 s R C}$ \\
\hline$\theta_{2 D H}$ & $-\frac{\bar{\pi}}{3}+\theta_{2 s: c}$ \\
\hline$\theta_{3 n H}$ & $\frac{\pi}{2}+\theta_{3 S R C}$ \\
\hline$\theta_{4 D H}$ & $\theta_{4 S R C}$ \\
\hline $0_{5 n H}$ & $\pi+\theta_{S_{S R C}}$ \\
\hline$\theta_{6-3}$ & $\overline{\frac{\pi}{2}}+\theta_{G_{S B C}}$ \\
\hline$\theta_{i, j i}$ & $\theta_{i_{S R C}}$ \\
\hline
\end{tabular}

Table A.2: Sarcos Slave arm: Joint limits

The sensors offset are shown in table A.1.2.

Table A.3: Corr: pondance between DH and SRC 


\section{A.2 Jacobian}

The Jacobian can be divided into two parts. First. $f_{l}$ represents the mappiug from joint angle velocities to translational velocities and can be obtained by dilferentiation of the end-effector position $(x, y, z)$. Second $J_{a}$. maps the joint angle velocities to angular velocities. It is obtained tising. the third column of the rotation mat rix from the base frame to the joint velocity being expressed.

$$
\begin{aligned}
& J_{l}=\left(\begin{array}{l}
\frac{d x}{d \theta} \\
\frac{d y}{d \theta} \\
\frac{d z}{d \theta}
\end{array}\right) \\
& J_{a}=\left(\begin{array}{lllllll}
{ }_{1}^{0} R_{z} & { }_{2}^{0} R_{z} & { }_{3}^{0} R_{z} & { }_{i}^{0} R_{z} & { }_{5}^{0} R_{z} & { }_{6}^{0} R_{z} & { }_{i}^{0} R_{z}
\end{array}\right) \\
& J=\left(\begin{array}{c}
J_{l} \\
J_{a}
\end{array}\right) \\
& j_{11}=-\left(l_{1} s_{1} s_{2}\right)+l_{2}\left(-\left(c_{1} s_{1} s_{2}\right)+\left(-\left(c_{2} c_{3} s_{1}\right)+c_{1} s_{3}\right) s_{1}\right) \\
& j_{12}=c_{1} c_{2} l_{1}+l_{2}\left(c_{1} c_{2} c_{1}-c_{1} c_{3} s_{2} s_{4}\right) \\
& j_{13}=l_{2}\left(c_{3} s_{1}-c_{1} c_{2} s_{3}\right) s_{1} \\
& j_{14}=l_{2}\left(c_{4}\left(c_{1} c_{2} c_{3}+s_{1} s_{3}\right)-c_{1} s_{2} s_{4}\right) \\
& j_{15}=0 \\
& j_{16}=0 \\
& j_{17}=0 \\
& j_{21}=c_{1} l_{1} s_{2}+l_{2}\left(c_{1} c_{4} s_{2}+\left(c_{1} c_{2} c_{3}+s_{1} s_{3}\right) s_{4}\right) \\
& j_{22}=c_{2} i_{3} s_{1}+l_{2}\left(c_{2} c_{4} s_{1}-c_{3} s_{1} s_{2} s_{4}\right) \\
& j_{23}=l_{2}\left(-\left(c_{1} c_{3}\right)-c_{2} s_{1} s_{3}\right) s_{4} \\
& j_{24}=l_{2}\left(c_{1}\left(c_{2} c_{3} s_{1}-c_{1} s_{3}\right)-s_{1} s_{2} s_{4}\right)
\end{aligned}
$$




$$
\begin{aligned}
& j_{25}=0 \\
& j_{2 t i}=0 \\
& j_{2:}=0 \\
& j_{31}=0 \\
& j_{32}=l_{1} s_{2}+l_{2}\left(c_{1} s_{2}+c_{2} c_{3} s_{4}\right) \\
& j_{33}=-\left(l_{2} s_{2} s_{3} s_{4}\right) \\
& j_{34}=l_{2}\left(c_{3} c_{4} s_{2}+c_{2} s_{4}\right) \\
& j_{35}=0 \\
& j_{3 i}=0 \\
& j_{3 \pi}=0 \\
& j_{41}=s_{1} \\
& j_{12}=c_{1} s_{2} \\
& j_{43}=c_{3} s_{1}-c_{1} c_{2} s_{3} \\
& j_{44}=c_{1} c_{4} s_{2}+\left(c_{1} c_{2} c_{3}+s_{1} s_{3}\right) s_{4} \\
& j_{45}=c_{5}\left(c_{3} s_{1}-c_{1} c_{2} s_{3}\right)-\left(c_{4}\left(c_{1} c_{2} c_{3}+s_{1} s_{3}\right)-c_{1} s_{2} s_{4}\right) s_{5} \\
& j_{46}=-\left(c_{6}\left(-\left(c_{1} c_{4} s_{2}\right)-\left(c_{1} c_{2} c_{3}+s_{1} s_{3}\right) s_{4}\right)\right)+\left(c _ { 5 } \left(c_{4}\left(c_{1} c_{2} c_{3}+s_{1} s_{3}\right)\right.\right. \\
& \left.\left.-c_{1} s_{2} s_{4}\right)+\left(c_{3} s_{1}-c_{1} c_{2} s_{3}\right) s_{5}\right) s_{6} \\
& j_{4 \pi}=c_{6}\left(-\left(c_{1} c_{4} s_{2}\right)-\left(c_{1} c_{2} c_{3}+s_{1} s_{3}\right) s_{4}\right) \\
& -\left(c_{5}\left(c_{1}\left(c_{1} c_{2} c_{3}+s_{1} s_{3}\right)-c_{1} s_{2} s_{4}\right)+\left(c_{3} s_{1}-c_{1} c_{2} s_{3}\right) s_{5}\right) s_{6} \\
& j_{51}=-c_{1} \\
& j_{52}=s_{1} s_{2} \\
& j_{53}=-\left(c_{1} c_{3}\right)-c_{2} s_{1} s_{3} \\
& j_{54}=c_{4} s_{1} s_{2}+\left(c_{2} c_{3} s_{1}-c_{1} s_{3}\right) s_{4} \\
& j_{55}=c_{5}\left(-\left(c_{1} c_{3}\right)-c_{2} s_{1} s_{3}\right)-\left(c_{4}\left(c_{2} c_{3} s_{1}-c_{1} s_{3}\right)-s_{1} s_{2} s_{4}\right) s_{5}
\end{aligned}
$$




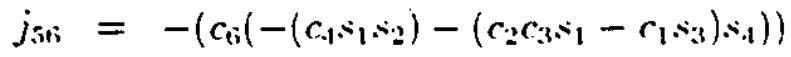

$$
\begin{aligned}
& +\left(c_{5}\left(c_{1}\left(c_{2} c_{3} s_{1}-c_{1} s_{3}\right)-s_{1} s_{2} s_{4}\right)+\left(-\left(c_{1} c_{3}\right)-c_{2} s_{1} \cdot s_{3}\right) s_{3}\right) s_{4} \\
& j_{57}=c_{6}\left(-\left(c_{1} s_{1} s_{2}\right)-\left(c_{2} c_{3} \cdot s_{1}-c_{1} \cdot s_{3}\right) \cdot s_{1}\right)-\left(c _ { 3 } \left(c_{4}\left(c_{2} c_{3} \cdot *_{1}-c_{1} \cdot s_{3}\right)\right.\right. \\
& \left.\left.-s_{1} s_{2} s_{4}\right)+\left(-\left(c_{1} c_{3}\right)-c_{2} s_{1} s_{3}\right) s_{5}\right) s_{6} \\
& j_{61}=0 \\
& j_{62}=-c_{2} \\
& j_{63}=-\left(s_{2} s_{3}\right) \\
& j_{6, i}=-\left(c_{2} c_{4}\right)+c_{3} s_{2} s_{4} \\
& j_{65}=-\left(c_{5} s_{2} s_{3}\right)-\left(c_{3} c_{4} s_{2}+c_{2} s_{4}\right) s_{5} \\
& j_{66}=-\left(c_{6}\left(c_{2} c_{4}-c_{3} s_{2} s_{4}\right)\right)+\left(c_{5}\left(c_{3} c_{4} s_{2}+c_{2} s_{4}\right)-s_{2} s_{3} s_{5}\right) s_{6} \\
& j_{6 t}=c_{6}\left(c_{2} c_{4}-c_{3} s_{2} s_{4}\right)-\left(c_{5}\left(c_{3} c_{4} s_{2}+c_{2} s_{1}\right)-s_{2} s_{3} s_{5}\right) s_{4}
\end{aligned}
$$

\section{A.2.1 Jacobian Extension}

Redundant manipulators have a rectangular Jacobian matrix but it is possible to extend it to produce a square matrix.

We decided to add an additional constraint to the Cartesian coordinates in order to take advantage of the available redundant degree of freedom. The angle between the $Z$ axis of the base frame and the axis of the elbow joint. We have,

$$
\begin{aligned}
x_{\text {elbow }} & =l_{1} c_{1} s_{2} \\
y_{\text {elbow }} & =l_{1} s_{1} s_{2} \\
z_{\text {elbow }} & =-l_{1} c_{2} \\
x_{\text {end-efsector }} & =l_{1} c_{1} s_{2}+l_{2}\left(c_{1} c_{4} s_{2}+\left(c_{1} c_{2} c_{3}+s_{1} s_{3}\right) s_{4}\right) \\
y_{\text {end-esfector }} & =l_{1} s_{1} s_{2}+l_{2}\left(c_{4} s_{1} s_{2}+\left(c_{2} c_{3} s_{1}-c_{1} s_{3}\right) s_{4}\right)
\end{aligned}
$$




$$
z_{\text {ruit-rsjector }}=-l_{1} c_{2}+l_{2}\left(-c_{2} c_{4}+c_{3} *_{2} ._{1}\right)
$$

The equation of the plane formed by those two vectors is,

$$
\begin{aligned}
\pi & =D c t\left(\begin{array}{ccc}
\vec{i} & \vec{j} & \vec{k} \\
x_{\text {elbow }} & y_{\text {elbow }} & z_{\text {elbow }} \\
x_{\text {end-cfsector }} & y_{\text {end-effector }} & z_{\text {end-efsector }}
\end{array}\right) \\
& =a \vec{i}+b \vec{j}+c \vec{k} \\
\text { normal } & =\left(\begin{array}{lll}
a & b & c
\end{array}\right)^{T} \\
Z & =\left(\begin{array}{lll}
0 & 0 & 1
\end{array}\right)^{T} \\
\text { normal } \cdot Z & =\sqrt{a^{2}+b^{2}+c^{2}} \cos \left(0_{\text {normal }}\right) \\
\theta_{\text {normal }} & =\cos ^{-1}\left(s_{2} s_{3}\right)
\end{aligned}
$$

Thus the additional constraint in the Jacobian matrix is expressed as a new line,

$$
\begin{aligned}
& j_{\text {new }}=0 \text { normal } \\
& j_{\text {new }}=\left(\begin{array}{llll}
0 & \frac{-\cos _{1}}{\sqrt{1-s_{2}^{2} 2_{3}^{2}}} & \frac{-s_{2} c_{2}}{\sqrt{1-s_{2}^{2} s_{3}^{2}}} & 0
\end{array}\right)
\end{aligned}
$$

Given $\theta_{\text {nornal }}$ it is possible to select an impedance for this new constraint as we do for the other Cartesian coordinates directions.

\section{A.3 Inverse Kinematics}

Finding the inverse kinematics of a redundant manipulator is usually not a simple task since there are multiple solutions for the position and orientation. Depending on the manipulator architecture, the problem may simplify. The Sarcos (GRLA) arm has its last three axes intersecting at one point. The position of the point of intersection 
depends only on the first four joint angles. A simple way to find a solution to the inverse kinematics problem is to fix one of the joint angles and then lind the position as a function of the three remaining joint angles. Once the first four joints are knowt:. Pieper's solution is used [3].

Assuming that $O_{3}$ is fixed by the user (or any optimization criterion exploiting the redundancy of the manipulator) we find. using equations (A.10). (A.11) and (A.12).

$$
\begin{aligned}
& 0_{4}= \pm \cos ^{-1}\left(\frac{x^{2}+y^{2}+z^{2}-l_{1}^{2}-l_{2}^{2}}{2 l_{1} l_{2}}\right) \\
& \theta_{3}=\text { fixed by the user } \\
& \theta_{2}=\operatorname{Atan} 2\left(l_{2} c_{3} s_{4},-\left(l_{1}+l_{2} c_{4}\right)\right) \pm \operatorname{Atan} 2\left(\frac{\sqrt{l_{2}^{2} c_{3}^{2} s_{4}^{2}+\left(l_{1}+l_{2} c_{1}\right)^{2}-z^{2}}}{z}\right) \\
& \theta_{1}=\operatorname{Atan} 2(x,-y) \pm \operatorname{Atan} 2\left(\frac{\sqrt{x^{2}+y^{2}-l_{2}^{2} s_{3}^{2} s_{4}^{2}}}{l_{2} s_{3} s_{4}}\right)
\end{aligned}
$$

the last three joint angles are found using the desired orientation matrix $R_{0}^{\pi}$ and the orientation given by the previously found joint angles, $R_{0}^{4}$ :

$$
R_{4}^{\bar{\tau}}=R_{0}^{1} R_{\bar{\tau}}^{0}=\left(\begin{array}{lll}
r_{11} & r_{12} & r_{13} \\
r_{21} & r_{22} & r_{23} \\
r_{31} & r_{32} & r_{33}
\end{array}\right)
$$

also

$$
R_{4}^{\bar{i}}\left(R_{6}^{\bar{\tau}}\right)^{-1}=R_{\mathrm{i}}^{\mathrm{j}} R_{5}^{\mathrm{i}}
$$

leading to

$$
\left(\begin{array}{ccc}
c_{5} c_{6} & -s_{5} & c_{5} s_{6} \\
s_{5} c_{6} & c_{5} & s_{5} s_{6} \\
-s_{6} & 0 & c_{6}
\end{array}\right)=\left(\begin{array}{ccc}
r_{11} c_{i}+r_{12} s_{i} & r_{11} s_{i}-r_{12} c_{i} & -r_{13} \\
r_{21} c_{i}+r_{22} s_{i} & r_{21} s_{i}-r_{22} c_{i} & -r_{23} \\
r_{31} c_{i}+r_{32} s_{i} & r_{31} s_{i}-r_{32} c_{\bar{i}} & -r_{33}
\end{array}\right)
$$

From the above matrices, we find directly $\theta_{5}$,: 


$$
0_{5}=\operatorname{Atan} 2\left(-r_{23},-r_{13}\right)
$$

then 0 :

$$
0_{6}=\operatorname{Atan} 2\left(-r_{13} c_{5}-r_{23} s_{5},-r_{33}\right)
$$

finally,

$$
\begin{aligned}
& -s_{5}=r_{11} s_{\bar{T}}-r_{12} c_{\bar{t}} \\
& c_{5}=r_{21} s_{\bar{\tau}}-r_{22} c_{\bar{i}}
\end{aligned}
$$

thus

$$
\begin{aligned}
& s_{7}=\frac{r_{22} s_{5}+r_{12} c_{5}}{r_{12} r_{21}-r_{11} r_{22}} \\
& c_{7}=\frac{r_{21} s_{5}+r_{11} c_{5}}{r_{12} r_{21}-r_{11} r_{22}}
\end{aligned}
$$

and

$$
\theta_{i}=\operatorname{Atan} 2\left(s_{i}, c_{i}\right)
$$

The inverse kinematics solution of this manipulator leads to eight different configurations since $\theta_{1}, \theta_{2}, \theta_{3}$ and $\theta_{4}$ have each two different possible values. 


\section{Appendix B}

\section{Calibration}

In the last chapters we presented a simple theory and supported it with simulations and experimentation. The calibration of the sensors and analog circuits is explained here. Figure B.I shows the organization of the controller used on cach joint of the Sarcos GRLA manipulator.

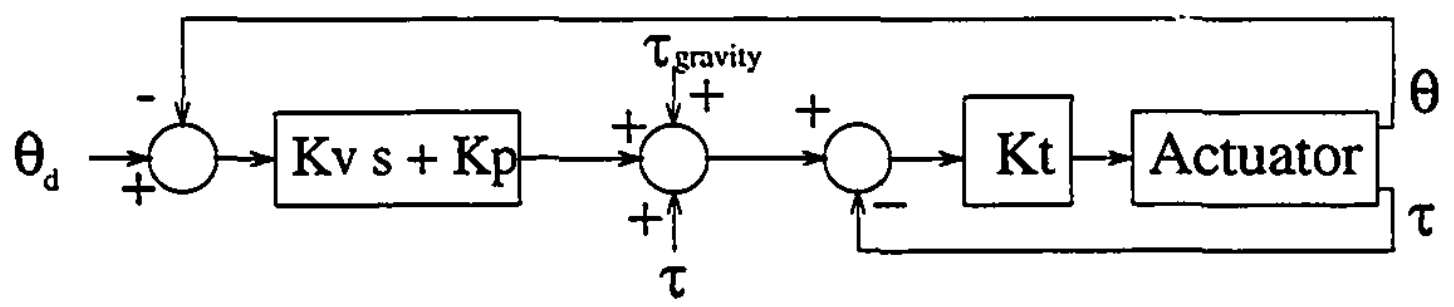

Figure B.1: Analog Joint Controller card

\section{B.1 Proportional and Derivative controller gains}

The decentralized impedance control implementation uses the PD controller gains to select the joint space diagonal terms of the stiffness and damping matrix. The off diagonal terms are used to form a cross-coupling torque to be substracted as a torque 
bias. We need to find the conversion factors from machine units to physical units $(\mathrm{Nm} / \mathrm{rad}$ and $\mathrm{Nm} /(\mathrm{rad} / \mathrm{scc}))$.

\section{B.1.1 Proportional term}

The correspondance between the voltage measurement and the joint angle (in radian) is given by equation B.1. The correspondance between the voltage measurement and the joint torque (in $\mathrm{Nm}$ ) is given in equation B.2.

$$
\begin{aligned}
\theta & =\frac{\pi}{20} v_{\theta} \\
\tau & =\frac{\text { Maximum load cell capacity }}{\mathrm{S}} v_{\tau} \\
\tau & =\frac{20}{\pi} \frac{\text { Maximum load cell capacity }}{\mathrm{S}}
\end{aligned}
$$

Table B.1.1 presents the results from an experiment conducted to check the nominal gain.

\begin{tabular}{|c|c|c|}
\hline Max. load $(\mathrm{Nm})$ & $G_{\text {thc }}(\mathrm{Nm} / \mathrm{rad})$ & $G_{\text {exp }}(\mathrm{Nm} / \mathrm{rad})$ \\
\hline 3443.11 & 2739.88 & 2672 \\
\hline 3443.11 & 2739.88 & 2648 \\
\hline 1411.11 & 1122.92 & 1112 \\
\hline 1411.11 & 1122.92 & 1112 \\
\hline 222.39 & 176.98 & 174.75 \\
\hline 254.0 & 202.13 & 195.82 \\
\hline 254.0 & 202.13 & 201.31 \\
\hline
\end{tabular}

Table B.1: Controller Proportional term calibration 


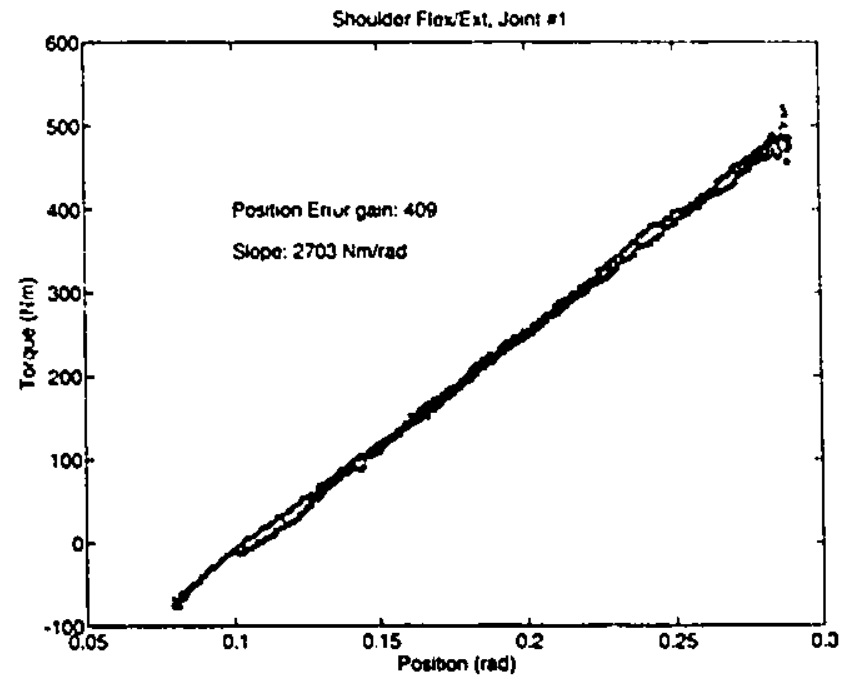

Figure B.2: Joint \#1 torque vs position

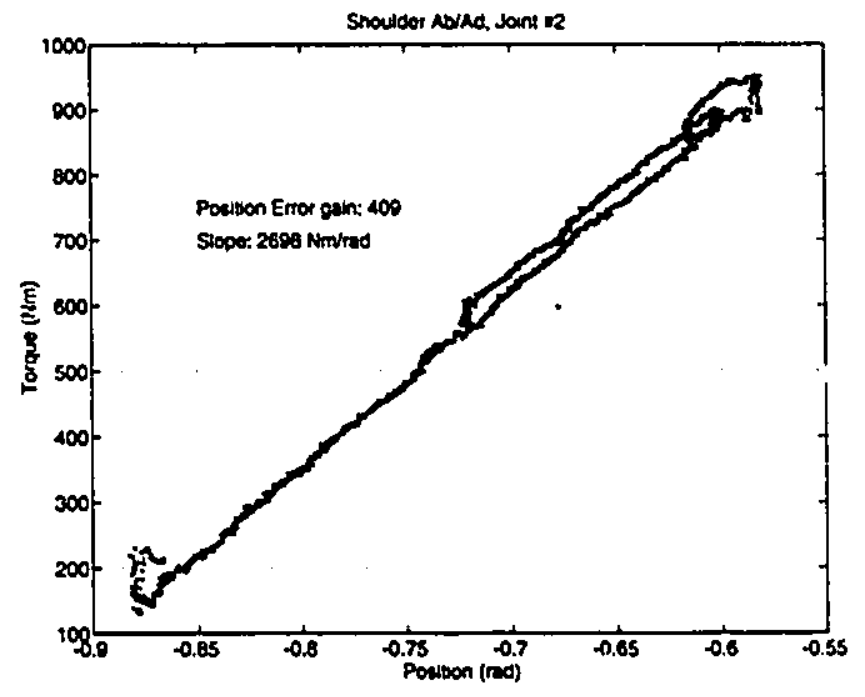

Figure B.3: Joint \# 2 torque vs position 


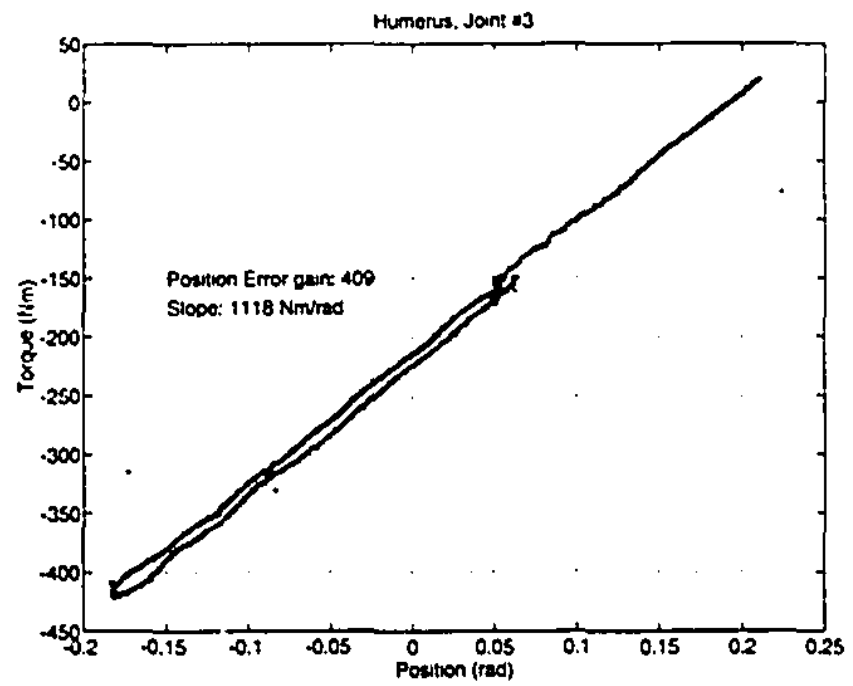

Figure B.4: Joint \# 3 torque vs position

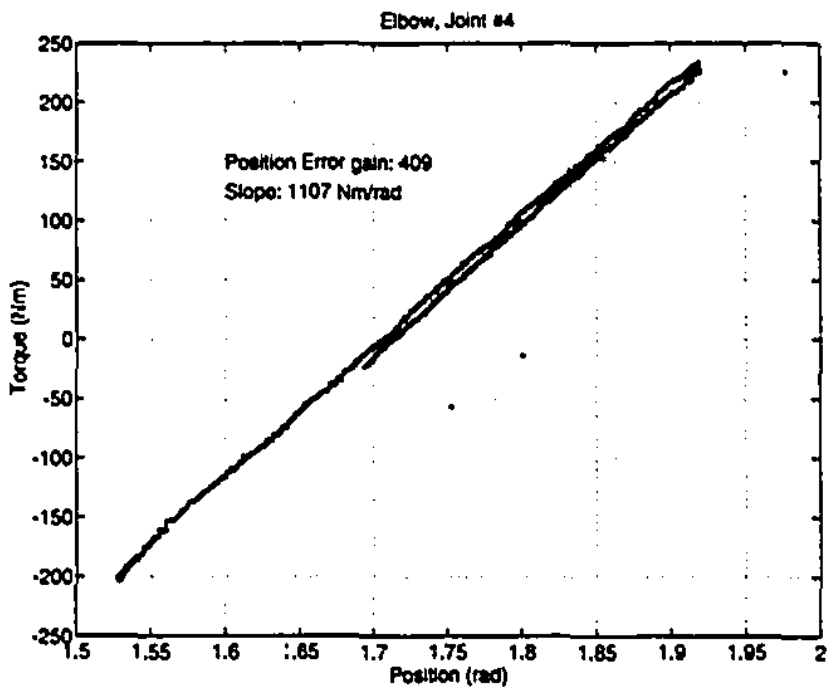

Figure B.5: Joint \# 4 torque vs position 


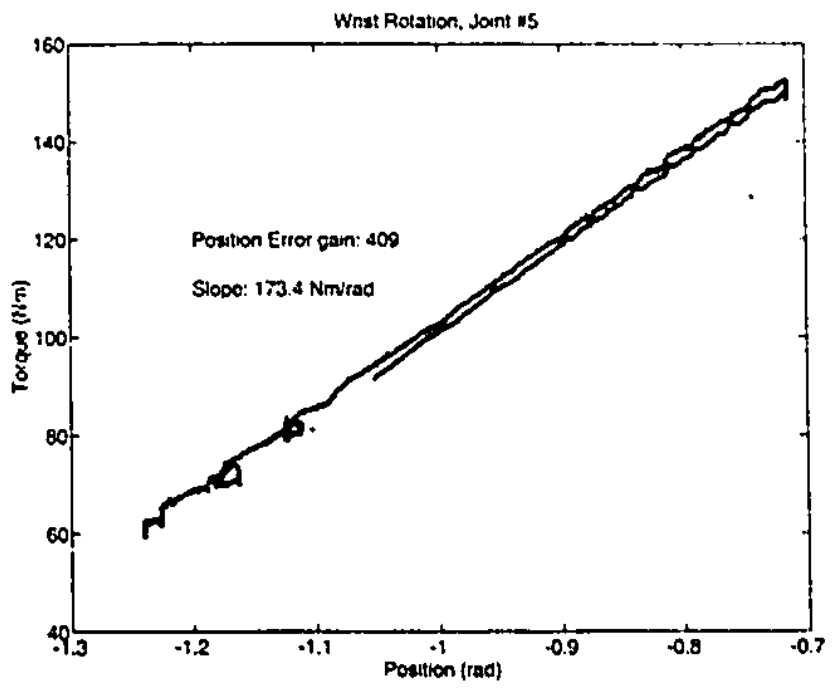

Figure B.6: Joint \# 5 torcue vs position

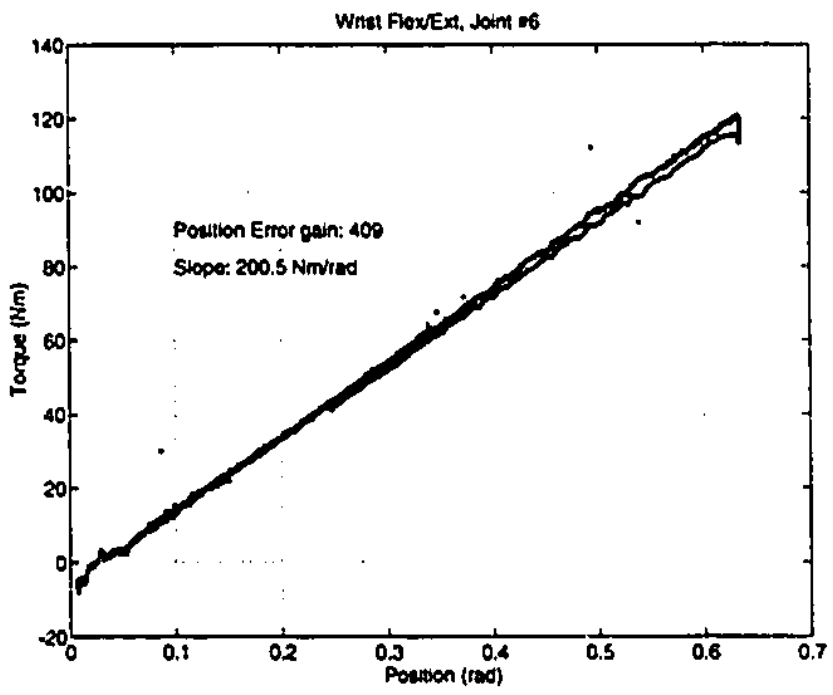

Figure B.T: Joint \# 6 torque vs position 


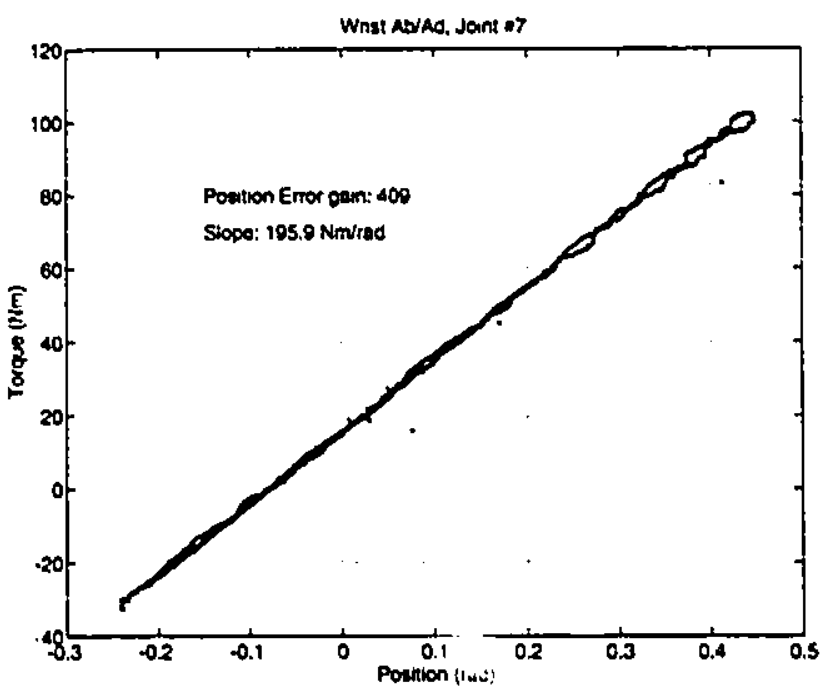

Figure B.S: Joint \# 7 torque vs position 


\section{B.1.2 Derivative term}

The analog joint controller cards include an anaiog differentiator estimating the velocity of each joint. Its transfer function (ser figure 13.9) is:

$$
\frac{\dot{X}}{X}=\frac{R_{2} C_{1} s}{\left(R_{2} C_{2} s+1\right)\left(R_{1} C_{1} s+1\right)}
$$

where $R_{1}=5.23 k \Omega, C_{1}=0.1 \mu f, R_{2}=511 k \Omega . C_{2}=0.001 \mu f$.

The differentiator attenuates high frequencies at the rate of $20 \mathrm{~d} / 3 / \mathrm{dec}$ ade. Figure B.10, shows its Bode ploi. For frequencies lower than $1912 \mathrm{rad} / \mathrm{sec}(300 \mathrm{Il} \%)$. Equation B.5 reduces to:

$$
\frac{\dot{X}}{\dot{X}}=R_{2} C_{1} s=0.0511 \mathrm{~s}
$$

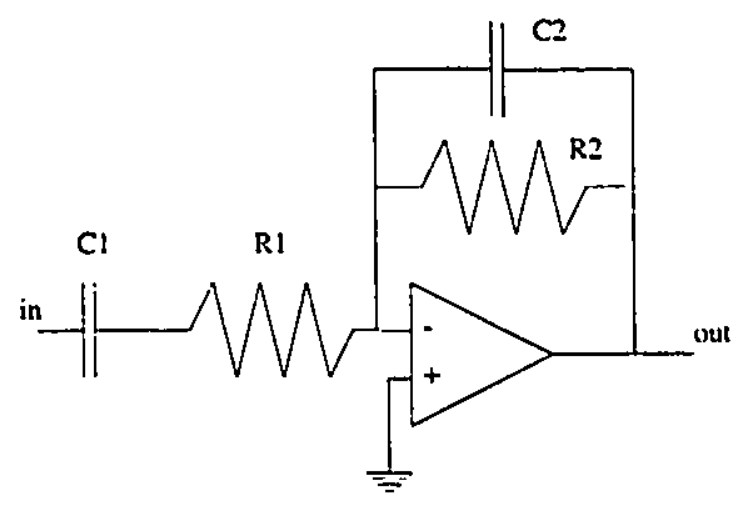

Figure B.9: differentiator circuit

The maximum voltage that can be supplied by the differentiator is 10 volts, corresponding to a velocity of $30.74 \mathrm{rad} / \mathrm{sec}$. This is two order of magnitude below the cutoff frequency of the differentiator and corresponds to extremcly high velocitics.

To compute the proper conversion factor from volts to $\mathrm{rad} / \mathrm{sec}$ we use the method described in section B.1.1

$$
\dot{\theta}=\frac{\pi}{20 R_{2} C_{1}} v_{\dot{\theta}}
$$



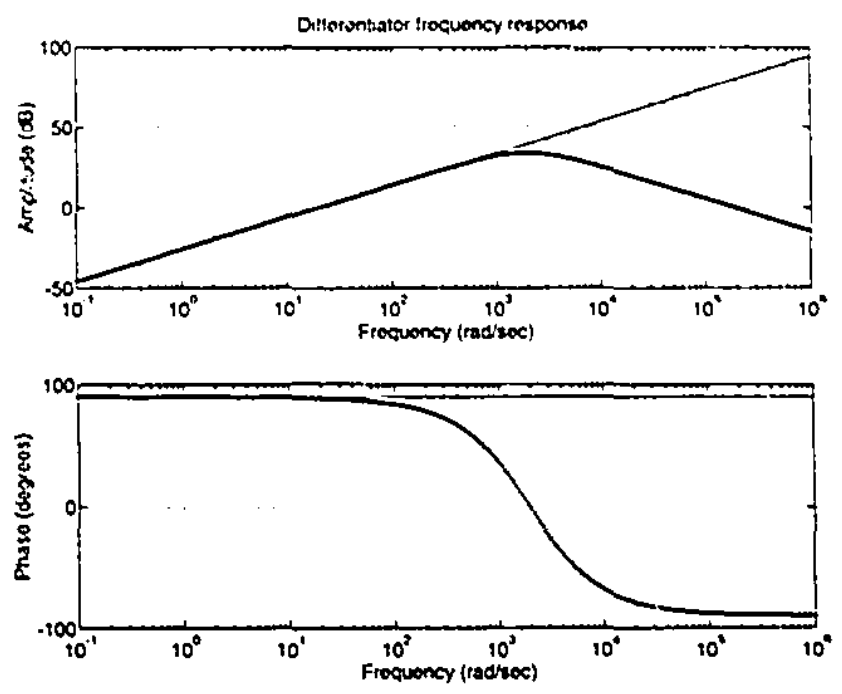

Figure B.10: differentiator transfer function

$$
\begin{aligned}
\tau & =\frac{\text { Maximum load cell capacity }}{\mathrm{s}} r_{\tau} \\
\frac{\tau}{\dot{\phi}} & =\frac{20 R_{2} C_{1}}{\pi} \frac{\text { Maximum load cell capacity }}{\$} \\
20 \text { volts } & \equiv \frac{\pi}{R_{2} C_{1}} \\
\mathrm{~S} \text { volts } & \equiv \text { maximum load cell capacity } \\
\mathrm{Nm} /(\mathrm{rad} / \mathrm{sec}) & =\frac{20 R_{2} C_{1}}{\pi} \frac{\text { max load cell capacily }}{\mathrm{S}}
\end{aligned}
$$

Then from equation B.12 we can find in table B.1.2 the proper correspondance for each joint. 


\begin{tabular}{|c|c|}
\hline Max. load $(\mathrm{Nm})$ & $G_{\text {the }}(\mathrm{Nm} /(\mathrm{rad} / \mathrm{sec}))$ \\
\hline 30500 & 140.01 \\
\hline 30500 & 140.01 \\
\hline 12500 & 57.35 \\
\hline 12500 & 57.35 \\
\hline 1970 & 9.04 \\
\hline 2000 & 10.33 \\
\hline 2000 & 10.33 \\
\hline
\end{tabular}

Table B.2: Controller Derivative term calibration 


\section{B.2 From Joint Torques to Cartesian Forces}

In non redundant robots. the inverse Jacobian matrix is used to derive the end-clfector wrench from the torques $\left(F=J^{-T}\right)$. When dealing with redundant manipulators. the Jacobian matrix cannot be inverted since it is not square. One approach is to neglect one row of the Jacobian and compute the corresponding force with a reduced set of joint torques. One criterion is to eliminate the line corresponding to:

$$
\min _{r}\left(\sum_{i}\left|J_{r, i}\right|\right)
$$

A pseudo inverse method may also be used to minimize the cror between $\left|\tau-J^{T} F\right|$.

$$
F=\left(J J^{T}\right)^{-1} J \tau
$$

Yet another method is to add one line to the Jacobian to make it square and then inverts it. The additional line lies in the space created by the redundant degree of freedom. 


\section{Bibliography}

[1] An C.H. and Hollerbach J.M. (198T). Kinematic Stability Issues in Force Control of Manipulators. IEEE International Conference on Robotics and Automation, pp. $\$ 97-903$.

[2] Asada H. and Slotine J.-J.E. (1986). Robot Analysis and Control, WileyIntersciencc.

[3] Craig J.J. (1989). Introduction to Robotics, Mechanics and Control, second edtion, Addison Wesley.

[4] Craig J.J. and Raibert M. (1979). A Systematic Method for Hybrid Position/Force Control of a Manipulator. Proceedings of the 1979 IEEE Computer Software Applications Conference, pp.446-451.

[5] Chen C.T. (1984). Linear System Theory and Design, second edition, HRW, pp. $168-231$.

[6] Dawson D.M., Lewis F.L. and Dorsey J.F. (1992). Robust Force Control of a Robot Manipulator. The International Journal of Robotics Research, 11(4):312319.

[7] Doyon M., Hayward V. and Pelletier M. (1994). Decentralized Impedance Control. Procedings of the 1994 IFAC Symposium on Robot Control, Capri, Italy. 
[8] Duffy J. (1990). The Fallacy of Modern Hybrid Control Theory that is Based on "Orthogonal Complements" of Twist and Virench Spaces. Jourmal of Robotics Systcms, $i(2): 139-1.4$.

[9] Eppinger S.D. and Secring W.P. (1986). On Dynamic Models of Robot Force Control. IEEE Intcrnational Conference on Robotics and A tutomation. pp. 29 34.

[10] Eppinger S.D. and Seering W.P. (19Si). Understanding Bandwidth Limitations in Robot Force Control. IEEE International Conference on Robotics and Atutomation, pp. 904-909.

[11] Eppinger S.D. and Seering W.P. (1989). Three Dynamic problems in Robot Force Control. IEEE International Conference on Robotics and Aulomalion, pp. 392397 .

[12] Fisher W.D. and Mujtaba M.S. (1992). Hybrid Position/Force Control: A Correct Formulation. The International Journal of Robolics Rescarch, 11(4):299-311.

[13] Field G. and Stepanenko Y. (1992). Robot Impedance Control without a Dynamic Model: a Model Reference Approach. IASTED International Confcrence on Control and Robotics, pp. 110-113.

[14] Franklin G. F., Powell J. D. and Workman M. L. (1990). Digital Control of Dynamic Systems, second edition, Addison-Wesley.

[15] Goldenberg A.A. (1992). Analysis of Force Control Based on Linear Modeis. IEEE International Conference on Robotics and Automation, pp. 1348-1353.

[16] Hayward V., Nemri C., Chen X. and Duplat B. (1993). Hand Controller Based on a Parallel Mechanism With Decoupled Stiffness. Journal of Robotics Systerns, 10(5): $767-790$. 
[17] Hogan N. (1985). Impedance Control: An Approach to Manipulation part I. II. 111. Journal of Dynamic Systcms. Measurement and Control. 107:1-24.

[18] Huang II.P. and Chen S.S. (1992). Compliant Motion Control of Robots by using Variable Impedance. International Journal of Advanced Manufacturing Tcchnol$o g y, 7: 322-332$.

[19] Iwaki S. and Matsuo S. (1990). A New Approach to Compliance Control of Robot Manipulators using Stable Negative Compliance. Robotics Research 1990. Winter Annual Meeting of the American Society of Mechanical Engineering, pp. $145-156$.

[20] Kancko M., Inamura N., Yokoi K. and Tanie K. (198S). Direct Compliance Control of Manipulator Arms - Basic Concept and Application Examples. Proceedings of 1988 IFAC Symposium on Robot Control, pp. S.1-8.6.

[21] Khatib O. (19ST). A Unified Approach for Motion and Force Control of Robot Manipulators: The Operational Space Formulation. IEEE Journal of Robotics and Automation, RA-3(1)43-53.

[22] Khorrami F. and Ozguner U. (19SS). Decentralized Control of Robot Manipulators via State and Proportional-Intregral Feedback. IEEE International Conference on Robotics and Automation, pp. 1198-1203.

[23] Lawrence D.A. (19SS). Impedance Control Stability Properties in Common Implementations, IEEE International Conference on Robotics and Automation, pp. $1155-1190$.

[24] Maples J.A., Becker J.J. (1986). Experiments in Force Control of Robotic Manipulators. IEEE Intcrnational Conference on Robotics and Automaticn, pp. 695702. 
[25] Pelletier M.. Doyon M. (1994). On the Implementation and Performance of Impedance Control on Position Controlled Robots. IREE International ('onfercnce on Robotics and Automation. pp. 1225-12:33.

[26] Peng Z. X. and Adachi N. (1993). Compliant Motion Control of Kinematically Redundant Manipulators. IEEE Transactions on Robotics and A utomation. $9(6): 831-837$.

[27] Salisbury J.K. (i980). Active Stiffness Control of a Manipulator in Cartesian Coordinates. Proceedings 19th IEEE Conference on Decision and Control. pp. 95-100.

[2S] Slotine J.J.E. and Li W. (1987). On the Adaptive Control of Robot. Manipulators. International Journal of Robotics Rescarch, 6(3):49-59.

[29] Strang G. (19S6). Introduction to applied mathematics. Wellesley-Cambridge Press.

[30] Tanie K., Yokoi K. and Kaneko M. (1990). Compliance Control with Consideration of Trajectory Control Characteristics. Procecdings of the 1990 ASME International Computers in Engineering Conference and Exposition, pp. 275280 .

[31] Yokoi K. (19SS). A Compliance Control Method Suggested by Muscle Networks in Human Arms. Proceedings of the 1988 IEEE International Workshop on Intelligent Robots and Systems, pp. 385-391.

[32] Whitney D.E. (1987). Historical Perspective and State of the Art in Robot Force Control. The International Journal of Robotics Research, 6(1):3-14. 
[33] Wu (... and Paul R.P. (1980). Manipulator Compliance Baseci on Joint Torque Control. Procedings of the 19th IEEE Conference on Decision and Control. 1:S894.

[31] Thang H. (1989). Kinematic Stability of Robot Manipulators under Force Control. IEEE International Conference on Robotics and Atutomation. pp. S0-\$5.

[35] Ziren L. and Goldenberg A.A. (1992). Implementation of Robust Impedance and Force Control. Journal of Intclligent and Robotic Systcms, 6:145-16:3. 